\title{
Network pharmacology, molecular docking, and experimental study for the mechanisms of Qishen Yiqi Pills against Cardiovascular Diseases
}

Jie-wen Zhao

Department of critical care medicine, the First Affiliated Hospital of Harbin Medical University

Hai-dong Liu

Capital Medical University Youan Hospital

\section{Ming-yin Man}

Department of critical care medicine, the first affiliated hospital of harbin medical university

\section{Lv-ya Wang}

Capital Medical University Affiliated Anzhen Hospital

Ning Li

Capital Medical University Youan Hospital

Jing Zhao

Shanghai University of Traditional Chinese Medicine

Wen-li Cheng ( $\nabla$ chengwenli2020@ccmu.edu.cn)

Department of Hypertension, Beijing Anzhen Hospital, Capital Medical University

\section{Kai-jiang Yu}

Department of critical care medicine, the First Affiliated Hospital of Harbin Medical University

\section{Research}

Keywords: Cardiovascular diseases, Qishen Yiqi Pills, Network pharmacology, targets, Hypoxia/reoxygenation injury

Posted Date: September 15th, 2021

DOI: https://doi.org/10.21203/rs.3.rs-889464/v1

License: (c) (i) This work is licensed under a Creative Commons Attribution 4.0 International License. Read Full License 


\section{Abstract \\ Background}

Qishen Yiqi Pills (QSYQP) is a traditional Chinese compound recipe. However, our understanding of its mechanism has been hindered due to the complexity of its components and targets. In this work, the network pharmacologybased approaches were used to explore QSYQP's pharmacological mechanism on treating cardiovascular diseases (CVD).

\section{Results}

From ETCM and TCM MESH databases we collected QSYQP's 333 active components and their 674 putative targets. We constructed the sub-network influence by CVD genes and found that 40\% QSYQP targets appeared in 20 modules, in which QSYQP's targets and CVD genes co-existed as hub nodes in the sub-network. Functional enrichment analysis suggested that the 42 key targets were mainly expressed in platelets, blood vessels, cardiomyocytes, and other tissues. The main signaling pathways regulated and controlled by the key targets were inflammation, immunity, blood coagulation and energy metabolism. Network and pathway analysis identified 7 key targets, which were regulated by 7 compounds of QSYQP. 26 of the 42 important targets, including the 7 key targets were verified by literature mining. Twelve pairs of interactions between key targets and QSYQP's compounds were validated by molecular docking. Further validation experiments suggested that QSYQP suppressed H/R induced apoptosis and cytoskeleton disruption of cardiomyocytes. Western blotting showed that the expression of cardiovascular diseases-related genes including ACTC1, Fox01 and DIAPH1 was significantly decreased by establishing the hypoxia-reoxygenation model in vitro, while the protein expression of experimental group was significantly increased by adding QSYQP or its ingredients.

\section{Conclusion}

These results indicated the correlation of QSYQP treatment to the therapeutic effects of CVD. At the molecular level, this study revealed the multicomponent and multitargeting mechanisms of QSYQP in the regulation and treatment of cardiovascular diseases, potentially providing a reference for the further utilization of QSYQP.

\section{Background}

Cardiovascular diseases (CVD) are a class of diseases affecting the heart or blood vessels, which mainly include coronary artery disease, peripheral vascular disease, congestive heart failure, hypertension, dyslipidemia, etc. As a main cause of death, cardiovascular diseases are associated with a "high incidence rate, high recurrence rate, high lethality rate, high disability rate" and "multiple complications". It has been reported that $80 \%$ of global cases of cardiovascular diseases will occur in low-and middle-income countries by the end of $2020^{[1]}$. In China, CVD has become the leading cause of disease-related death ${ }^{[2]}$. Therefore, seeking more effective cardiovascular drugs to reduce the health-related economic burden of these diseases is urgently needed ${ }^{[3]}$.

The Chinese compound recipe Qishen Yiqi Pills (hereinafter called QSYQP) has a significant effect on cardiovascular diseases including preserving microvascular function in patients undergoing percutaneous coronary 
intervention and secondary prevention of myocardial infarction ${ }^{[4 ; 5]}$. The application of QSYQP for the treatment of CVD was approved by the Chinese Food and Drug Administration.

The incidence and mortality of coronary heart disease is high, which leads to a significant increase in healthcare and economic burden, the burden of which is increasing. The conventional western medicine treatment can be implemented as soon as possible to save lives. However, there is no effective treatment in western medicine for coronary microcirculation angina after treatment of myocardial infarction. Coronary microvascular dysfunction is characterized as typical angina but exhibits normal coronary arteries angiograms ${ }^{[6]}$. For the myocardial fibrosis and disturbance in the microcirculation caused by ischemia reperfusion injury after coronary intervention therapy, Chinese medicine has showed little side effects and unique advantages ${ }^{[7]}$. For example, a large sample multicenter study on secondary prevention of coronary heart disease showed that QSYQP had the same therapeutic effect as aspirin in secondary prevention of myocardial infarction, and it was less likely to cause gastrointestinal complications $^{[5]}$.

Although modern medicine has made certain achievements in the treatment of cardiovascular diseases, these treatments may cause a series of side effects. Earlier studies have found that QSYQP could inhibit myocardial fibrosis caused by ischemia-reperfusion injury $[8 ; 9]$, especially relieve the myocardial hypertrophy caused by hypertension ${ }^{[10 ; 11]}$. TCM formulas, the main effects of which are tonifying Qi, promoting blood circulation, and removing blood stasis, have achieved remarkable effects in treating cardiovascular diseases ${ }^{[9]}$. With multicomponent, multitarget, and synergistic effects ${ }^{[12]}$, Chinese herbal medicines are safer and more efficacious as well as less toxic and associated with fewer side effects than other drugs; hence, Chinese herbal medicine have gradually become a hot topic in recent research ${ }^{[13 ; 14]}$.

There are four Chinese herbs in the compound recipe QSYQP: Astragalus membranaceus (Huang Qi,HQ), Salvia miltiorrhiza (Dan Shen, DS), Panax notoginseng (San Qi, SQ), and Dalbergia odorifera (Jiang Xiang, JX). Salvia miltiorrhiza is one of the most common Chinese herbs in activating blood circulation and removing blood stasis. Panax notoginseng, belonging to Araliaceae, can regulate the tension of blood vessels, such as by dilating blood vessels, increasing blood circulation, eliminating blood stasis, and inhibiting platelet adhesion. Dalbergiae odoriferae, as a traditional Chinese medicine, is the dried heartwood of the trunk and root of a leguminous plant. Its main components are volatile oil and flavonoids. Astragalus membranaceus comes from the root of a perennial plant in the pea family, with a sweet taste and warm nature. It supplements Qi and elevates Yang, consolidates superficies, prevents sweating, drains pus, expels toxins, induces diuresis, alleviates edema, and promotes tissue regeneration.

Some earlier studies have focused on 17 bioactive compounds from QSYQP herbs to explore their mode of action ${ }^{[15]}$. Gene expression experiments were performed in a rat model of myocardial infarction treated with QSYQP and illustrated the potential targets and anti-inflammatory effects of QSYQP ${ }^{[16]}$. Ultra-performance liquid chromatography coupled with triple quadrupole mass spectrometry was applied to identify 19 bioactive compounds in QSYQP ${ }^{17]}$. These studies provide a good basis for further investigation of QSYQP's mechanisms in the treatment of CVDs.

Network pharmacology, as a new interdisciplinary science, can study the laws and mechanisms of drug action at a system level ${ }^{[3 ; 18]}$. It integrates the techniques of many disciplines including pharmacology, systems biology, and computational biology to explore the relationship between drugs and diseases in general, and provides new ideas 
and perspectives for the study of complex effects of drugs ${ }^{[12 ; 19]}$. In this study, we collected compounds of QSYQP, their potential targets and pathways to verify the further protective effect and mechanism of QSYQP. On hypoxia/reoxygenation injury in $\mathrm{H} 9 \mathrm{c} 2$ cardiomyocytes, we observed the repair of cellular cytoskeleton and the inhibition of apoptosis, and also detected the expression of proteins in myocardial contraction related pathways, including ACTC1, Fox01, DIAPH1. This work may offer a reference for the future utilization of QSYQP or drug repositioning by exploring the effects and mechanisms of QSYQP in the treatment of cardiovascular diseases.

\section{Materials And Methods}

\subsection{Collecting ingredients and predicting targets}

The active constituents and potential targets in the formula were identified by searching the names of Chinese herbal medicine "HUANG QI", "DAN SHEN", "SAN QI", and "JIANG XIANG", in ETCM ${ }^{[20]}$ and TCM-Mesh database ${ }^{[21]}$ respectively. ETCM (http://www.nrc.ac.cn: 9090 / ETCM /) is a database containing information of 403 Chinese herbal medicines, 7274 ingredients, and 2266 targets. TCM-Mesh database (http://mesh.tcm.microoinformatics.org/) contains the information of more than 700 herbs and integrates a variety of information resources.

\subsection{Constructing the sub-network influenced by cardiovascular diseases}

Taking "cardiovascular diseases" as the keyword and searching DisGeNET database v6. $0^{[22]}$ (https://www.disgenet.org/), the genes related to cardiovascular diseases were obtained. Based on the high-quality protein-protein interaction network of human genome in the HINT ${ }^{[23]}$ (High-quality INTeractomes) database (http://hint.yulab.org/), the genes related to cardiovascular diseases was taken as seeds. The seeds as well as their neighbor nodes and connected edges between these nodes were extracted from the background network to constructed the sub-network influenced by cardiovascular diseases. Cytoscape software was used in the construction and analysis of the sub-network ${ }^{[24]}$.

\subsection{Analyzing the functional module of the sub-network}

The module analysis of the sub-network influenced by cardiovascular diseases was based on the method of module analysis of protein-protein interaction networks, using the Molecular Complex Detection (MCODE) app of Cytoscap $\mathrm{e}^{[24]}$. MCODE is a clustering algorithm based on graph theory, which can quickly detect the densely connected domain in large-scale protein networks, and rate the degree of protein association in the module. Through the Database for Annotation, Visualization, and Integrated Discovery (DAVID) ${ }^{[25 ; 26]}$ and the STRING ${ }^{[27 ; 28]}$ platform (https://string-db.org/ version 11.0), the Gene Ontology (GO) functional enrichment analysis of the submodule was performed and the multiple biological pathways of the drug in treating diseases were systematically revealed. DAVID can describe and evaluate the target from multiple perspectives, such as disease enrichment analysis, tissue enrichment analysis, and so on. STRING was used to perform the functional enrichment of the concerned genes.

\subsection{Identifying important targets and conducting functional enrichment analysis}


In the sub-network influenced by cardiovascular diseases, the drug target proteins with the degree more than twice of the average degree were searched and taken as important targets. DAVID was used to conduct the disease and tissue enrichment analysis to clarify the targets' function.

\subsection{KEGG pathway analysis of important targets}

The STRING platform was used to carry out the KEGG pathway analysis of important targets. The pathway information was obtained by mapping the acquired important targets to the KEGG pathway ${ }^{[29]}$ (KEGG, http://www.genome.jp/kegg/, updated on January 14, 2020). KEGG was developed in Japan and based on the pathway data obtained from the Kyoto Encyclopedia of Genes and Genomes database.

\subsection{Molecular docking}

The crystal structures of protein receptors were downloaded from RCSB Protein Data Bank (PDB) database ${ }^{[30]}$. The chemical structures of compounds were extracted from PubChem database ${ }^{[31]}$. The original binding ligands and other molecules, such as waters or metals, were removed by PyMol ${ }^{[32]}$. Protein receptors and ligands were then prepared by AutoDock Tools ${ }^{[3]}$. The binding site of the original ligand with the protein receptor was specified as the docking site of the protein. Finally, each compound was docked into the protein receptors using AutoDock Vina ${ }^{[33]}$.

\subsection{Hypoxia-reoxygenation injure of $\mathrm{H} 9 \mathrm{c} 2$ cardiomyocytes}

The H9c2 cells, were obtained from the National biomedical experimental cell resource bank (BMCR). QSYQP (Batch number:20091005) was acquired from Tasly Pharmaceutical Co. Ltd. The major components of QSYQP, danshensu (DSS) and astragaloside IV (ASIV) were purchased from Kunming Feng Shan Jian Medical Research Co. Ltd. Antibodies including Fox01 (18592-1-AP), ACTC1 (66125-1-lg), and DIAPH1 (20624-1-AP) were purchased from Proteintech Group Inc.

Cells were cultured in Dulbecco's Modified Eagle Medium (DMEM) (Gibco, Carlsbad) supplemented with 10\% foetal bovine serum (FBS) at $37^{\circ} \mathrm{C}$ in an incubator with a humidified atmosphere of $5 \% \mathrm{CO}_{2}$, and the medium was replaced every three days. Hypoxia and reoxygenation $(H / R)$ culture of cells: three gas incubators were used for hypoxia, the oxygen concentration was $1 \%$, and the culture was 24 hours. Then the oxygen recovery condition: turn to the normal incubator; the oxygen concentration is $21 \%$, culture for 12 hours, replace it with the normal medium. The cells were divided into six groups: Control group (Control), hypoxia and reoxygenation group (H/R), H/R + danshensu group (H/R + DSS), H/R + astragaloside IV group (H/R + ASIV), H/R + danshensu and astragaloside IV group (H/R + DSS + ASIV), H/R + Qishen Yiqi Pills group (H/R + QSYQP), After 8 hours incubation, cells were harvested for the examination of apoptosis, and cell Cytoskeleton.

\subsection{Determination of cell death by TUNEL assay}

Terminal deoxynucleotidyl transferase (TdT) nick-end labelling Cell apoptosis was analyzed using TUNEL kit from Nanjing Kaiji Biology (kga7071). Briefly, cells were washed with cold $1 \times$ PBS for three times, fixed with 4\% paraformaldehyde for 15 minutes and permeabilized by incubation in $1 \times$ PBS containing $0.1 \%$ Triton X-100 for 10 minutes at room temperature. Cells were then washed three times with $1 \times$ PBS and incubated with the labeling solution (containing fluorescein-dUTP) for 1 hour at $37^{\circ} \mathrm{C}$ in dark according to the manufacturer's instruction. Finally, nucleus was stained with DAPI. Apoptotic cells were observed using a fluorescence microscope (Leica, TCS SP8).

\subsection{Measurement of the (F- actin) cytoskeleton}


Each well was prefixed with $200 \mu \mathrm{L}$ medium $+200 \mu \mathrm{L} 4 \%$ paraformaldehyde for 5 min, and fixed with $400 \mu \mathrm{L} 4 \%$ paraformaldehyde for $10 \mathrm{~min}$. The solution was discarded and washed with PBS once, add $0.1 \%$ TritonX-100 (PBS dilution) to each well, room temperature for $10 \mathrm{~min}$. PBS wash 5 min 3 times, phalloidin 1:50 dilution (PBS) was incubated at room temperature for $30 \mathrm{~min}$, wash with PBS for $5 \mathrm{~min}, 2$ times, nucleus staining (dark room) 1:1000 was used by diluted DAPI in dark at room temperature incubation for 7-10min, PBS washing for 3 times, and taken photos with laser confocal microscope.

\subsection{Western blotting assay}

Cells were lysed in cold RIPA buffer after experiments, and protein was separated by centrifugation. BCA Protein Assay Kit (Sigma-Aldrich) was used for the determination of protein concentration. Loading buffer was added to the cytosolic extracts and then heated on at $100^{\circ} \mathrm{C}$ for 5 minutes; the same amounts of supernatant from each sample were subjected to $10 \%$ or $12 \%$ sodium dodecyl sulphate-polyacrylamide gel electrophoresis (SDS-PAGE), and then the total number of proteins was transferred onto PVDF membranes. The membranes were blocked overnight in $5 \%$ fat-free milk at $4^{\circ} \mathrm{C}$ and incubated with their respective antibodies, including ACTC1, Fox01, DIAPH1 and GAPDH monoclonal antibodies at the recommended diluted concentration, then visualized by anti-rabbit or anti-mouse horseradish peroxidase-conjugated secondary antibody (1:1000) and finally developed with ECL chemiluminescence detection reagent (Amersham Pharmacia Biotech). In order to quantify the protein expression levels, the X-ray films were scanned and analyzed using Image J software (Wayne Rasband, NIH).

\section{Results}

\subsection{Chemical compounds and their targets in the formula}

By using the ETCM and TCM-Mesh databases, 333 active compounds (Supplementary Table 1) in QSYQP were identified, among which 158 compounds were found to have 674 corresponding targets (Supplementary Table 2), including 40 active compounds of Astragalus membranaceus and their 507 corresponding targets, 47 active ingredients of Salvia miltiorrhiza and 337 corresponding targets, 65 active constituents of Panax notoginseng and 382 related targets, as well as 22 active components of Dalbergia odorifera and their 122 corresponding targets. There were 674 different targets in the formula after removing the redundancies. The numbers of targets in Astragalus membranaceus, Salvia miltiorrhiza, Panax notoginseng, and Dalbergia odorifera were 507, 337, 382, and 122, respectively. See Fig. 1 for the compound-target networks of the four medicines. Figure 2 shows the herb-target network of the formula. It can be seen that many targets were influenced by multiple herbs, suggesting the synergistic effects of the formula.

The overlaps between ingredients and targets of the four herbs in QSYQP are shown in Fig. 3. The four herbs had no common components, but they had 69 common targets. Salvia miltiorrhiza, Panax notoginseng, and Dalbergia odorifera had 87 common targets. Astragalus membranaceus, Salvia miltiorrhiza, and Panax notoginseng had 209 targets in common. Astragalus membranaceus and Panax notoginseng are more powerful when working together, which could prove the synergistic mechanism of Jun, Chen, Zuo, Shi (the principal, assistant, complement, and guide drug in TCM formula) in TCM theory.

\subsection{Subnetwork influenced by cardiovascular diseases genes}

Using the DisGeNET v6.0 (https://www.disgenet.org/, updated in May 2019) database and inputting "cardiovascular diseases" as the keyword, 786 genes related to cardiovascular diseases were obtained (Supplementary Table 3). The high-quality protein-protein interaction network of human genome in the HINT database was used as the 
background network. Networks are transmissible, and the influences on the network nodes will first transmit to their adjacent genes. In networks, the genes that are first influenced by disease genes are their neighbors. Taking the 786 genes related to cardiovascular diseases as the seeds and extracting the neighboring genes of the seeds in the background network, the interaction network of the genes was constructed with Cytoscape 3.6 software. Removing the small connected components with only a few nodes and retaining the largest one, the subnetwork influenced by cardiovascular diseases was constructed, which contained 3942 nodes and 26230 edges.

\subsection{Analysis of the effects of QSYQP on the disease subnetwork}

The 674 targets of QSYQP were mapped to the sub-network influenced by cardiovascular diseases genes. The targets mapped to the sub-network were the genes by which the drug components affect the disease. The more the targets were mapped to the disease sub-network, the greater the drug's effect on the disease was. In this subnetwork, 275 of the 674 targets were mapped, accounting for $40.8 \%(275 / 674=40.8 \%)$ of targets, in which 125 nodes were both cardiovascular diseases genes and the targets of QSYQP (see Fig. 4).

The MCODE algorithm was used to conduct the module analysis of the subnetwork influenced by cardiovascular diseases. Setting k-core = 2, 45 modules could be obtained in total, and the degree of each node was at least 2; 20 modules could be identified that contained target proteins and at least 3 nodes. See Fig. 7 for the modules containing target proteins.

The biological process involved in each module was analyzed. The functional enrichment analysis of each cluster shows that each module had significant biological function. For example, Module 5 contained 56 genes influenced by cardiovascular diseases. DAVID tissue enrichment analysis of the targets in the module showed that platelets and vascular endothelium were the main targets of drug action. The pathways involved were the PI3K-Akt signaling pathway, EGFR tyrosine kinase inhibitor resistance, the Ras signaling pathway, and the MAPK signaling pathway. Module 7 contained 79 genes and was mainly enriched in the relaxin signaling pathway, the atherosclerosis signaling pathway, the PI3K Akt signaling pathway, the HIF-1 signaling pathway and platelet activation. This module played a particularly important role in the connection between cells, participating in the adjustment of adherens junctions, gap junctions, and tight junctions, in the process of exudation, leakage, edema, leukocyte adhesion to endothelial cells, etc. Module 11 contained 79 genes and focused on inflammation and apoptosis. In terms of pathways, it was involved in the NF-KB signaling pathway, the apoptosis-related signaling pathway, the TNF signaling pathway, fluid shear stress and atherosclerosis, the toll-like receptor signaling pathway, etc. The pharmacodynamic mechanism of Module 8 and Module 18 focused on the regulation of inflammation and immunity.

\subsection{The important targets of QSYQP}

The average degree of the subnetwork influenced by cardiovascular diseases is 13.3. Taking the targets with a degree greater than 27 as hub nodes, 42 important targets of QSYQP were obtained in total, and 18 of them were genes relevant to cardiovascular diseases.

The associated network of herb-compound-target for the important targets of QSYQP is shown in Fig. 6A. The network was constructed using Cytoscape software, including 4 herbs, 42 important targets, 97 compounds, and 530 interactions. Approximately one-third of the compounds of QSYQP target 42 important targets, suggesting synergetic effects of the formula on cardiovascular diseases. 
Then we used STRING platform to construct protein-protein interaction network between the important targets, in which the confidence score of links were set as at least 0.9 (Fig. 6B). We used Cytoscape software to visualize and analyze the network. The nodes with degree, betweenness and closeness larger than corresponding medium values were considered as hubs of the network, which may play more important roles. In this way, 15 hub nodes were obtained, i.e., TP53, EP300, PIK3CA, PIK3R1, HSP90AA1, ESR1, CREBBP, AKT1, SRC, NR3C1, NFKB1, AR, CCND1, NFKBIA, EGFR.

\subsection{Functional enrichment analysis of important targets}

Functional enrichment analysis of diseases and tissues of the important targets was carried out with the DAVID v6.8 database to identify the main diseases and target tissues that the important targets acted on.

\section{1) Disease enrichment analysis}

Via the "GAD-DISEASE" database in the DAVID platform, six clusters were obtained by conducting the disease enrichment analysis of the 42 important targets in the network and setting the classification stringency to "highest" (Table 1). All of the clusters with enrichment score that ranked in the top three covered cardiovascular diseases and their related diseases. This indicated that the important targets played a role in treating cardiovascular diseases. The higher the enrichment score was, the more enriched the target.

Table 1

Disease enrichment of important genes.

\begin{tabular}{|c|c|c|c|}
\hline Rank & $\begin{array}{l}\text { Enrichment } \\
\text { score }\end{array}$ & Disease term & $\begin{array}{l}\mathrm{P} \text { - } \\
\text { value }\end{array}$ \\
\hline \multirow[t]{3}{*}{$\begin{array}{l}\text { Cluster } \\
1\end{array}$} & \multirow[t]{3}{*}{5.88} & $\begin{array}{l}\text { Cholesterol, HDL cholesterol, LDL fatty acid glucose insulin lipoprotein } \\
\text { triacylglycerols }\end{array}$ & $\begin{array}{l}5.82 \mathrm{E}- \\
07\end{array}$ \\
\hline & & Cardiovascular diseases risk & $\begin{array}{l}1.62 \mathrm{E}- \\
06\end{array}$ \\
\hline & & cognitive impairment & $\begin{array}{l}2.43 \mathrm{E}- \\
06\end{array}$ \\
\hline \multirow[t]{4}{*}{$\begin{array}{l}\text { Cluster } \\
2\end{array}$} & \multirow[t]{4}{*}{3.81} & colon cancer; rectal cancer & $\begin{array}{l}2.92 \mathrm{E}- \\
05\end{array}$ \\
\hline & & androgen levels & $\begin{array}{l}9.69 \mathrm{E}- \\
05\end{array}$ \\
\hline & & Cardiomyopathy, Hypertrophy, Left Ventricular & $\begin{array}{l}1.45 \mathrm{E}- \\
04\end{array}$ \\
\hline & & Biliary calculi|Biliary Tract Neoplasm|Biliary Tract Neoplasms|Gallstones & $\begin{array}{l}1.45 \mathrm{E}- \\
04\end{array}$ \\
\hline \multirow[t]{4}{*}{$\begin{array}{l}\text { Cluster } \\
3\end{array}$} & \multirow[t]{4}{*}{3.43} & Diabetes Mellitus, Type 1|Diabetes Mellitus, Type 2|Diabetic Retinopathy & $\begin{array}{l}3.75 \mathrm{E}- \\
05\end{array}$ \\
\hline & & Albuminuria|Inflammation|Kidney Diseases & $\begin{array}{l}6.42 \mathrm{E}- \\
05\end{array}$ \\
\hline & & Recurrence|Venous Thromboembolism & $\begin{array}{l}8.02 \mathrm{E}- \\
04\end{array}$ \\
\hline & & Brain Ischemia|Hypertension|Osteoporosis|Stroke & $\begin{array}{l}1.06 \mathrm{E}- \\
03\end{array}$ \\
\hline
\end{tabular}




\section{2) Tissue enrichment analysis}

To explore whether the target proteins of QSYQP acted on the target tissues of cardiovascular diseases, tissue enrichment analysis of the 42 key targets was conducted using the DAVID platform. As a result, 212 pieces of information on tissue enrichment were obtained, and these target proteins were mainly enriched in target tissues such as platelets, the vasculature, the vascular endothelium, the heart, cardiac myocytes, and the blood. (Table 2). Therefore, QSYQP plays a therapeutic role in cardiovascular diseases by acting on the target tissues of CVD.

Table 2

Tissue enrichment of important tissues.

\begin{tabular}{|llll|}
\hline Category & term & $\begin{array}{l}\text { P- } \\
\text { value }\end{array}$ & Mapped genes \\
\hline UP_TISSUE & Platelet & $\begin{array}{l}2.21 \mathrm{E}- \\
04\end{array}$ & $\begin{array}{l}\text { ACTB, TTR, APP, HSP90AA1, PRKAA1, NOS3, YWHAE, } \\
\text { SRC }\end{array}$ \\
\hline CGAP_EST_QUARTILE & $\begin{array}{l}\text { Vascular } \\
\text { normal }\end{array}$ & 0.0042 & ACTB, PIK3CA, NR3C1, YWHAE \\
\hline CGAP_SAGE_QUARTILE & $\begin{array}{l}\text { Vascular } \\
\text { endothelium }\end{array}$ & 0.0394 & ACTB, AR, CCND1, HSP90AA1, PIK3CA, SRC, SF3B3 \\
\hline UP_TISSUE & Heart & 0.0407 & HSP90AA1, PPARG, WNK1, PRKAA2, YWHAE \\
\hline UP_TISSUE & Epithelium & 0.0742 & $\begin{array}{l}\text { AKT1, EGFR, HSP90AA1, EP300, ERBB2, CREBBP, } \\
\text { CSNK2B, WNK1, ABL1, INSR, PIK3R1 }\end{array}$ \\
\hline
\end{tabular}

\subsection{KEGG pathway enrichment analysis of important targets}

KEGG pathway ${ }^{[29]}$ enrichment analysis of 42 important targets was conducted on STRING (FDR $<10^{-5}$ ), and 47 pathways related to cardiovascular diseases affected by the important targets were identified (Supplementary Table 4). The results showed that the biological processes involved in the key targets in the treatment of cardiovascular diseases focused on inflammation, immunity, coagulation, antioxidation, and blood pressure stabilization.

To elucidate the relationship between the proteins and the biological processes in which they are involved, 30 pathways with more than 4 mapping targets and FDR scores below $10^{-3}$ were selected. OmicShare tool, a free online platform for data analysis (http://www.omicshare.com/tools), was used to display the KEGG pathway enrichment of the important targets in four dimensions, and the bubble chart is shown in Fig. 7A.

Then we constructed target-pathway network between the pathways in Fig. 7A and important targets involved in these pathways (Fig. 7B). A total of 7 targets (AKT1, PIK3CA, PIK3R1, NFKB1, EGFR, SRC, CCND1) were involved in at least 10 pathways, suggesting that they could play more important roles in QSYQP's treatment to cardiovascular diseases. In addition, all of the 7 targets were hubs of protein-protein interaction network between the 42 important targets (Fig. 6B). Therefore, they were considered as key targets of QSYQP.

Next, we extracted all the compounds which interacted with the key targets and constructed the compound-target network for key targets of QSYQP (Fig. 7C). In this network, a total of 7 compounds (kaempferol, hexadecanoicacid, aceticacid, pentadecanoicacid, tanshinaldehyde, quercetin, luteolin) interacted with at least 2 key targets, suggesting that they could be key compounds of QSYQP for treating CVD. 


\subsection{Validation of important targets by literature mining and molecular docking}

We searched the drug-likeness grading of the 97 compounds which putatively targeted on the 42 important targets of QSYQP in the ETCM database. The drug-likeness gradings of 12, 22 and 63 compounds were good, moderate and weak, respectively. This result suggested that about one third of these compounds might show drug activities.

We then searched PubChem database to explore experimental evidences for the 42 important targets. By searching CID code for each of the 97 compounds in PubChem, 26 of the 42 important targets were found to be targeted by 47 of the compounds from the data in the "Chemical-Genes Co-Occurrence in Literature" section of PubChem, in which 13 validated targets were also CVD disease genes and 17 compounds had good or moderate drug-likeness gradings. In addition, it was validated by literature that all of the 7 key targets were targeted by QSYQP's compounds (see Fig. 7). Next, we input the 26 validated targets into the STRING platform to perform functional enrichment analysis. It was found that 37 KEGG pathways with more than 5 mapping targets were enriched with the input genes $\left(\mathrm{FDR}<10^{-5}\right), 34$ of which were also enriched with the 42 important targets. These results suggested the reliability of our analysis from the 42 important targets.

At last, We used AutoDock Vina software to perform molecular docking for the validation of the binding between QSYQP's key targets and the corresponding compounds. We downloaded the chemical structures of the 7 key compounds (kaempferol, hexadecanoicacid, aceticacid, pentadecanoicacid, tanshinaldehyde, quercetin, luteolin) (Fig. 7C) from PubChem database. The crystal structures of 6 targets interacting with these 7 compounds (Fig. 7C), i.e., AKT1 (PDB ID: 6buu), CCND1 (PDB ID: 2w96), EGFR (PDB ID: 5d41), PIK3CA (PDB ID: 6gvf), PIK3R1 (PDB ID: 1h9o), and SRC (PDB ID: 5k9i), were downloaded from RCSB Protein Data Bank. The docking score denotes the affinity between the compound and the target, i.e. the lower docking score suggests the better affinity. As shown in Table 3, the docking scores between 12 pairs of compound-target are lower than $-4 \mathrm{kcal} / \mathrm{mol}$, indicating better binding affinity between corresponding compounds and targets. In Fig. 8 we show binding poses of 6 pairs of compound-target interactions.

Table 3

The docking scores between QSYQP's key targets and compounds (kcal/mol).

\begin{tabular}{|lllllll|}
\hline Target/Compound & kaempferol & luteolin & quercetin & aceticacid & hexadecanoicacid & tanshinaldehyde \\
\hline AKT1 & -6.1 & -6.1 & -6.1 & $/$ & $/$ & $/$ \\
CCND1 & $/$ & -6.7 & $/$ & $/$ & $/$ & $/$ \\
\hline EGFR & $/$ & $/$ & -9.1 & $/$ & $/$ & $/$ \\
PIK3CA & $/$ & $/$ & $/$ & -6.7 & -6.4 & -11.1 \\
\hline PIK3R1 & $/$ & $/$ & $/$ & -4.8 & -4.3 & -7.6 \\
\hline SRC & -9.5 & $/$ & $/$ & $/$ & $/$ & $/$ \\
\hline
\end{tabular}

\subsection{QSYQP suppressed H/R induced cell apoptosis}

The impact of QSYQP on apoptosis of H9c2 cardiomyocytes after H/R treatment was assessed by TUNEL assay. We found some morphological changes such as rippled or creased nuclear and apoptotic bodies in Fig. 9. Strong green fluorescence was visualized in H/R group, but when administration with QSYQP, significantly reversed the 
quantity and intensity of fluorescence in H9c2 cardiomyocytes induced by H/R. The results showed that QSYQP significantly prevent apoptosis induced by H/R.

\subsection{QSYQP protected against H/R-inducedcytoskeleton changes}

Cytoskeleton plays an important role in maintaining cell morphology, withstanding external forces and maintaining the order of internal structure. As shown in Fig. 10, exposure of H9c2 cardiomyocytes to H/R caused a remarkable interrupt and exception aggregation, as evident by the significant confusion of red fluorescent signals in $\mathrm{H} / \mathrm{R}$ group compared to the normal condition. QSYQP significantly reversed the H/R-induced disruption of cytoskeleton.

\subsection{Target validation in cell-based studies}

To further verify the effect of QSYQP on cellular cytoskeleton and its binding proteins, we detected the expression of related proteins, including $\mathrm{ACTC1}$, Fox01, and DIAPH1. We conducted Western blot experiment in H9c2 cells. Compared with H/R group, the levels of ACTC1, Fox01, and DIAPH1 increased obviously after QSYQP and its main components' treatment (see Fig. 11).

\section{Discussion}

QSYQP was approved for the treatment of angina pectoris of coronary heart disease by China State Food and Drug Administration in 2003. Network pharmacology analysis is becoming an important research method to study the effect of Chinese medicines on diseases to reveal the complex components and unknown targets of Chinese medicines. Although QSYQP has been used in the clinic for a long time, its specific mechanism is not fully understood. Through ETCM and TCM-Mesh screening, 333 compounds were recognized as active compounds of QSYQP, 158 of which were predicted to act on 674 specific targets.

Overlap analysis of the targets of the four herbs in QSYQP and construction of the herb-target network revealed that most targets were jointly regulated by multiple compounds and herbs. Usually, the potency of a single natural compound's regulatory effect on a target is much lower than that of a specifically designed drug molecule because the interactions between natural compounds and proteins generally have low affinity. Our results suggest that QSYQP may produce therapeutic effects by acting on multiple proteins from multiple ingredients of different herbs to achieve synergetic efficacy.

An earlier network pharmacology study illustrated that the coexistence of disease genes and drug targets in the same network module suggested an underlying link between disease and drugs ${ }^{[34]}$. We constructed a subnetwork influence by CVD genes and found that 40\% of the targets of QSYQP appeared in this subnetwork, while 20 modules were identified in which target proteins and CVD genes coexisted. These results indicate that QSYQP may exhibit therapeutic effects on CVD.

By analyzing the topology of the subnetwork influenced by CVD, 42 targets of QSYQP were identified as key targets for the treatment of cardiovascular diseases. Furthermore, the important targets were found to be significantly enriched in 31 pathways, which were associated with the occurrence and development of cardiovascular diseases, including blood coagulation, inflammation, immunity and energy metabolism. KEGG pathway analysis identified that the important targets of QSYQP were involved in several pathways related to blood coagulation, such as complement and coagulation cascades, fluid shear stress and atherosclerosis, platelet activation, and the VEGF signaling pathway. Blood coagulation factors play important roles in cardiovascular diseases risk, participating in the initiation of the extrinsic coagulation process and causing the generation of thrombin ${ }^{[35-37]}$. A Previous 
publication indicated that Salvia miltiorrhiza ameliorated deep vein thrombosis by exerting antioxidative effects ${ }^{[38]}$. Coagulation factors regulated by 2-isopropyl-8-methylphenanthrene-3,4-dione extracted from Salvia miltiorrhiza were associated with complement and coagulation cascade signaling pathways. As part of the cardiovascular phenotype in congestive heart failure (CHF), platelet activation is correlated with impaired endogenous platelet inhibition and endothelial dysfunction ${ }^{[39]}$. Platelet activation is activated in atrial fibrillation (AF) and accelerates the progress of thrombin generation ${ }^{[40]}$. However, the level of VEGF is downregulated in the pathophysiology of heart failure ${ }^{[41]}$. Our results suggested that the active compounds JX, meliotocarpan c and odoricarpan, modulated the target TUBB. TUBB, which is specifically expressed by platelets and megakaryocytes, may be involved in the secretion and release of platelets ${ }^{[42]}$. QSYQP's targets ACTB, AKT1, NOS3, PIK3CA, PIK3R1, and SRC participated in platelet activation and VEGF signaling pathways, suggesting its function alleviating the symptoms of cardiovascular diseases by regulating blood coagulation.

The pathways correlated with inflammation and immunity, which are regulated by QSYQP, have also been identified, such as the PI3K-Akt signaling pathway, the Toll-like receptor signaling pathway (TLR), apoptosis, the HIF-1 signaling pathway, the T cell receptor signaling pathway, the chemokine signaling pathway, the TNF signaling pathway, the Jak-STAT signaling pathway, the Wnt signaling pathway, the Ras signaling pathway, the p53 signaling pathway, Th17 cell differentiation, the NF-kappa B signaling pathway, Th1 and Th2 cell differentiation, and the NOD-like receptor signaling pathway ${ }^{[43]}$. The NF-kappa B signaling pathway is associated with immune deficiency, whereas dysregulated activation of this pathway contributes to the pathogenesis of various autoimmune and inflammatory diseases. Our data show that QSYQP can participate in individual validation and immune regulation via the chemokine signaling pathway and NF-kappa B signaling pathway. As a serine threonine kinase, Akt participates in the processes of cell proliferation and survival and decreases necrosis and apoptosis of cardiomyocytes $^{[44-46]}$. Intracellular MAPK signaling cascades, probably play an important role in the pathogenesis of cardiovascular diseases ${ }^{[47]}$. The Toll-like receptor signaling pathway, which accelerates the nuclear translocation of NF-KB and AP-1, recruits TRIF, and activates the transcription of proinflammatory cytokine genes ${ }^{[48]}$.

In addition, pathways related to cardiovascular contraction, blood pressure regulation and energy metabolism have been identified, which could explain the mechanism of QSYQP in the treatment of cardiovascular diseases. Ten targets were mapped to adherens junctions, and nine were mapped to focal adhesions. The cell matrix adhered and participated in the structural connection between membrane receptors and the actin cytoskeleton, and played an important role in a series of biological processes. The cell matrix is connected with gap junction channels and is the basis of many physiological events, including electrical coupling, metabolic transport, apoptosis and maintenance of homeostasis. Eight targets participated in tight junctions, in which transmembrane protein composition is critical for building a diffusion barrier for selective infiltration between adjacent cells, affecting many biological pathways. In addition, the relaxin signaling pathway, which is involved in vasodilation related to proteins targeted by compound components, plays a key role in the regulation of blood pressure, aldosterone-regulated sodium reabsorption, and sodium and potassium metabolism. The proportion of oxidative energy supplied to myocardial free fatty acids will increase, and myocardial remodeling is the main pathological feature in the progression of myocardial ischemic injury or heart failure. Some studies have shown that disorder of myocardial energy metabolism directly or indirectly promotes the process of myocardial remodeling and further aggravates heart failure. A large number of studies have shown that cardiac hypertrophy is related to cardiac energy metabolism. QSYQP can reduce cardiac hypertrophy and improve cardiac function by regulating cardiac energy metabolism ${ }^{[49 ;}$ 50]. Multiple targets of the compound are mapped to the cGMP-PKG signaling pathway and cAMP signaling 
pathway, through which they improve and regulate myocardial energy metabolism, thus playing an important role in the treatment and symptom improvement of heart failure ${ }^{[51]}$.

To validate the results from network pharmacological analysis, we investigated the protective effect of QSYQP from myocardial cell injury using an H/R model in vitro. Our results strongly suggest the cardioprotective effect of QSYQP from H/R injury, which was mainly manifested on the decreases of cell apoptosis and protective effect of cytoskeleton. Exposure of $\mathrm{H} 9 \mathrm{c} 2$ cardiomyocytes to $\mathrm{H} / \mathrm{R}$ caused a remarkable interrupt and exception aggregation, as evident by the significant confusion of red fluorescent signals in $\mathrm{H} / \mathrm{R}$ group compared to the normal condition. However, H9c2 cardiomyocytes treated with QSYQP and its components showed a remarkable regulation.

At last, we applied Western blot experiment to validate the effects of QSYQP and its two active ingredients (DSS and ASIV) to three proteins, ACTC1, Fox01, and DIAPH1. Our network pharmacological analysis suggested that ACTC1 and DIAPH1 were targets of QSYQP, and FoxO1 was CVD disease gene. These three proteins were in the subnetwork influenced by CVD, as well as in important pathways associated with QSYSP's treatment. Specifically, ACTC1 is associated with Hypertrophic cardiomyopathy signaling pathway, DIAPH1 is associated with Regulation of actin cytoskeleton and Focal adhesion signaling pathway, FoxO1 is correlated with the AMPK signaling pathway. A remarkable increase in expression showed that ACTC1, Fox01, DIAPH1 might be involved in the cardiac injury induced by H/R. Our experiments showed that cardiomyocytes treated with QSYQP, DSS and ASIV had a remarkable increase in expression of ACTC1, Fox01, DIAPH1.

One of cardiovascular diseases is hypertrophic cardiomyopathy (HCM), a group of related diseases characterized by hypertrophy of the ventricular myocardium. It was thought to be the result of a particularly group of mutations in the cardiac actin gene ACTC1. A variety of genes linked to HCM encode proteins related to the sarcomere, the fundamental contractile unit of the heart, formed from interacting filaments of cardiac actin. Increasing evidence has shown that Fox01 is closely related to the occurrence and development of cardiac hypertrophy. FoxO1 can regulate cardiac growth, protein synthesis, calcium homeostasis, cell apoptosis, and autophagy. Fox01 undergoes modification and transfers from the cytoplasm to nucleus, thus regulating the expression of series of target genes in myocardium in response to stress or external stimulation. FOXO1 is an essential regulator of vascular growth in endothelium. Recent studies suggest that DIAPH1 is involved in the regulation of actin filaments. It has revealed that DIAPH1 rotates along the F-actin. Therefore, our experiments suggested that QSYQP regulated the three genes and associated pathways to play a therapeutic role for cardiovascular diseases.

These results ${ }^{[52-54]}$ may offer a reference for QSYQP's further utilization or the drug repositioning on new indications. Integrating network and pathway analysis, we identified 7 key targets of QSYQP in the treatment of cardiovascular diseases. Among the seven key targets, CCND1 is the target of AMPK pathway (hsa04152), which is related to cardiomyocyte hypertrophy ${ }^{[55]}$. SRC and EGFR are the targets of regulation of actin cytoskeleton (hsa04810) and play roles in the signal transduction of myocardial hypertrophy and alleviate myocardial hypertrophy ${ }^{[56]}$.

\section{Conclusions}

The targets of QSYQP corresponding to the chemical ingredients of Astragalus membranaceus, Salvia miltiorrhiza, Panax notoginseng, and Dalbergia odorifera can systematically treat cardiovascular diseases through multiple pathways. 
The formula increases myocardial contractility and improves myocardial energy metabolism through multiple components and multiple targets, which is in accord with the concept that Astragalus membranaceus is the predominant medicine that tonifies Qi. It has the potential to be applied for the treatment of chronic heart failure.

The targets of QSYQP are enriched in platelets, vascular endothelium, cardiomyocytes and other tissues and participate in pathways related to the coagulation cascade, such as intrinsic and extrinsic coagulation and platelet activation, to improve coagulation dysfunction in cardiovascular diseases, atherosclerosis, and inhibition of oxidative stress. This is in accordance with the concept of blood promotion in traditional Chinese medicine.

The formula treats cardiovascular diseases at a systematic level, and its treatment effect includes protection of the vascular endothelium, alterations in intercellular connections, inhibition of inflammation and apoptosis, stabilization of blood pressure, and effects on other multiple biological pathways. This process reflects the characteristics of the overall treatment and synergistic effects of traditional Chinese medicine formulas.

\section{Abbreviations}

QSYQP

Qishen Yiqi Pills

CVD

cardiovascular diseases

HQ

Huang Qi, Astragalus membranaceus

DS

Dan Shen, Salvia miltiorrhiza

SQ

San Qi, Panax notoginseng

$J X$

Jiang Xiang, Dalbergia odorifera

DSS

danshensu

ASIV

astragaloside IV

DMEM

Dulbecco's Modified Eagle Medium

FBS

foetal bovine serum

$\mathrm{H} / \mathrm{R}$

Hypoxia and reoxygenation

\section{Declarations}

\section{Availability of data and materials}

The relevant drug active compounds, disease targets and pathway names are available in the Supplementary Source files. 333 active compounds is in Supplementary Table 1; 674 corresponding targets is in Supplementary 
Table 2; 786 genes related to cardiovascular diseases were obtained in Supplementary Table 3; 47 pathways related to cardiovascular diseases is in Supplementary Table 4. Other datasets used and/or analysed during the study are available from the corresponding author upon request.

\section{Funding}

This study was supported by the National Natural Science Foundation of China [No. 81770276]. The funding source had no role in the design of the study and collection, analysis, and interpretation of the data and in writing the manuscript.

\section{Authors' contributions}

JWZ and JZ conception and design, interpretation of data, writing and revising and final approval of the manuscript submitted; MYM and HDL drafting of the manuscript; LYW and NL critical revision; KJY and WLC support of the study. All authors read and approved the final manuscript.

\section{Acknowledgements}

We also thank the OmicShare tool, a free online platform for data analysis (www.omicshare.com/tools/). We would also like to thank AJE (https://www.aje.com/go/springernature) for English language editing.

\section{Ethics approval and consent to participate}

Not applicable.

\section{Consent for publication}

Not applicable.

\section{Competing interests}

The authors declare no conflict of interests.

\section{References}

1. Roth GA, et al. Global and regional patterns in cardiovascular mortality from 1990 to 2013. Circulation. 2015;132(17):1667-78.

2. Liu S, et al. Burden of Cardiovascular Diseases in China, 1990-2016: Findings From the 2016 Global Burden of Disease Study. JAMA Cardiol. 2019;4(4):342-52.

3. Li S. Exploring traditional chinese medicine by a novel therapeutic concept of network target. Chin J Integr Med. 2016;22(9):647-52.

4. He GX, et al. Effects of Qishen Yiqi Dripping Pills in Reducing Myocardial Injury and Preserving Microvascular Function in Patients Undergoing Elective Percutaneous Coronary Intervention: A Pilot Randomized Study. Chin J Integr Med. 2018;24(3):193-9.

5. Shang $\mathrm{H}$, et al., Qi-shen-yi-qi dripping pills for the secondary prevention of myocardial infarction: a randomised clinical trial. Evid Based Complement Alternat Med, 2013. 2013: p. 738391.

6. Naderi S. Microvascular Coronary Dysfunction-an Overview. Curr Atheroscler Rep. 2018;20(2):7. 
7. Zheng QN, et al. QiShenYiQi Pills((R)) ameliorates ischemia/reperfusion-induced myocardial fibrosis involving RP S19-mediated TGFbeta1/Smads signaling pathway. Pharmacol Res. 2019;146:104272.

8. Lin SQ, et al. QiShenYiQi Pills(R) prevent cardiac ischemia-reperfusion injury via energy modulation. Int J Cardiol. 2013;168(2):967-74.

9. Han JY, et al. Effects and mechanisms of compound Chinese medicine and major ingredients on microcirculatory dysfunction and organ injury induced by ischemia/reperfusion. Pharmacol Ther. 2017;177:146-73.

10. Chen YY, et al. QiShenYiQi Pills, a compound in Chinese medicine, protects against pressure overload-induced cardiac hypertrophy through a multi-component and multi-target mode. Sci Rep. 2015;5:11802.

11. Li YC, et al. Attenuating effect of post-treatment with QiShen YiQi Pills on myocardial fibrosis in rat cardiac hypertrophy. Clin Hemorheol Microcirc. 2012;51(3):177-91.

12. Wang $Y$, et al. Strategies and techniques for multi-component drug design from medicinal herbs and traditional Chinese medicine. Curr Top Med Chem. 2012;12(12):1356-62.

13. Fang HY, et al. A network-based method for mechanistic investigation of Shexiang Baoxin Pill's treatment of cardiovascular diseases. Sci Rep. 2017;7:43632.

14. Gao L, et al. Uncovering the anticancer mechanism of Compound Kushen Injection against HCC by integrating quantitative analysis, network analysis and experimental validation. Sci Rep. 2018;8(1):624.

15. Zheng S, Zhang Y, Qiao Y, The Mechanism Research of Qishen Yiqi Formula by Module-Network Analysis. Evid Based Complement Alternat Med, 2015. 2015: p. 497314.

16. Li X, et al. A network pharmacology study of Chinese medicine QiShenYiQi to reveal its underlying multicompound, multi-target, multi-pathway mode of action. PLoS One. 2014;9(5):e95004.

17. Xie Q, et al. Simultaneous Determination of 19 Bioactive Constituents in QishenYiqi Dropping Pills by UltraPerformance Liquid Chromatography Coupled with Triple Quadrupole Mass Spectrometry. J AOAC Int. 2019;102(4):1102-11.

18. Li S, Zhang B. Traditional Chinese medicine network pharmacology: theory, methodology and application. Chin J Nat Med. 2013;11(2):110-20.

19. Li P, et al. Systems pharmacology strategies for drug discovery and combination with applications to cardiovascular diseases. J Ethnopharmacol. 2014;151(1):93-107.

20. Xu HY, et al. ETCM: an encyclopaedia of traditional Chinese medicine. Nucleic Acids Res. 2019;47(D1):D97682.

21. Zhang RZ, et al. TCM-Mesh: The database and analytical system for network pharmacology analysis for TCM preparations. Sci Rep. 2017;7(1):2821.

22. Bauer-Mehren A, et al. DisGeNET: a Cytoscape plugin to visualize, integrate, search and analyze gene-disease networks. Bioinformatics. 2010;26(22):2924-6.

23. Das J, Yu H. HINT: High-quality protein interactomes and their applications in understanding human disease. BMC Syst Biol. 2012;6:92.

24. Shannon P, et al. Cytoscape: a software environment for integrated models of biomolecular interaction networks. Genome Res. 2003;13(11):2498-504.

25. Huang DW, Sherman BT, Lempicki RA. Bioinformatics enrichment tools: paths toward the comprehensive functional analysis of large gene lists. Nucleic Acids Res. 2009;37(1):1-13. 
26. Huang DW, Sherman BT, Lempicki RA. Systematic and integrative analysis of large gene lists using DAVID bioinformatics resources. Nat Protoc. 2009;4(1):44-57.

27. Szklarczyk D, et al. STRING v11: protein-protein association networks with increased coverage, supporting functional discovery in genome-wide experimental datasets. Nucleic Acids Res. 2019;47(D1):D607-13.

28. Szklarczyk D, et al. The STRING database in 2021: customizable protein-protein networks, and functional characterization of user-uploaded gene/measurement sets. Nucleic Acids Res. 2021;49(D1):D605-12.

29. Kanehisa M, Goto S. KEGG: kyoto encyclopedia of genes and genomes. Nucleic Acids Res. 2000;28(1):27-30.

30. Burley SK, et al. RCSB Protein Data Bank: powerful new tools for exploring 3D structures of biological macromolecules for basic and applied research and education in fundamental biology, biomedicine, biotechnology, bioengineering and energy sciences. Nucleic Acids Res. 2021;49(D1):D437-51.

31. Kim S, et al. PubChem in 2021: new data content and improved web interfaces. Nucleic Acids Res. 2021;49(D1):D1388-95.

32. Alexander N, Woetzel N, Meiler J. bcl::Cluster: A method for clustering biological molecules coupled with visualization in the Pymol Molecular Graphics System. IEEE Int Conf Comput Adv Bio Med Sci. 2011;2011:138.

33. Tanchuk VY, et al, New A. Improved Hybrid Scoring Function for Molecular Docking and Scoring Based on AutoDock and AutoDock Vina. Chem Biol Drug Des. 2016;87(4):618-25.

34. Li H, et al., A network pharmacology approach to determine active compounds and action mechanisms of gegen-qin-lian decoction for treatment of type 2 diabetes. Evid Based Complement Alternat Med, 2014. 2014: p. 495840.

35. Alehagen U, Dahlstrom U, LindahI TL. Low plasma concentrations of coagulation factors II, VII and XI indicate increased risk among elderly with symptoms of heart failure. Blood Coagul Fibrinolysis. 2010;21(1):62-9.

36. Olson NC, et al. Associations of activated coagulation factor VII and factor VIla-antithrombin levels with genome-wide polymorphisms and cardiovascular disease risk. J Thromb Haemost. 2018;16(1):19-30.

37. Yamagishi $\mathrm{K}$, et al, Coagulation factors II, IX V, XI X, and XII, plasminogen, and alpha-2 antiplasmin and risk of coronary heart disease. J Atheroscler Thromb, 2010. 17(4): p. 402-9.

38. Cao H, et al. Salvia miltiorrhiza prevents deep vein thrombosis via antioxidative effects in endothelial cells. Mol Med Rep. 2015;11(5):3593-600.

39. Hacker A, et al. The nitric oxide donor pentaerythritol tetranitrate can preserve endothelial function in established atherosclerosis. Br J Pharmacol. 2001;132(8):1707-14.

40. Tarnowski $D$, et al. Comparison of diverse platelet activation markers as indicators for left atrial thrombus in atrial fibrillation. Platelets. 2018;29(1):41-7.

41. Taimeh Z, et al. Vascular endothelial growth factor in heart failure. Nat Rev Cardiol. 2013;10(9):519-30.

42. Caparros-Perez E, et al. Down Regulation of the Munc18b-syntaxin-11 Complex and beta1-tubulin Impairs Secretion and Spreading in Neonatal Platelets. Thromb Haemost. 2017;117(11):2079-91.

43. Zhou L, et al. QiShenYiQi Pills, a Compound Chinese Medicine, Prevented Cisplatin Induced Acute Kidney Injury via Regulating Mitochondrial Function. Front Physiol. 2017;8:1090.

44. Sadidi M, Lentz SI, Feldman EL. Hydrogen peroxide-induced Akt phosphorylation regulates Bax activation. Biochimie. 2009;91(5):577-85.

45. Teshima Y, et al. High-glucose condition reduces cardioprotective effects of insulin against mechanical stressinduced cell injury. Life Sci. 2010;87(5-6):154-61. 
46. Wang B, et al. Propofol protects against hydrogen peroxide-induced injury in cardiac H9c2 cells via Akt activation and Bcl-2 up-regulation. Biochem Biophys Res Commun. 2009;389(1):105-11.

47. Takeshima $\mathrm{H}$, et al. Cardioprotective effect of a combination of Rho-kinase inhibitor and p38 MAPK inhibitor on cardiovascular remodeling and oxidative stress in Dahl rats. J Atheroscler Thromb. 2012;19(4):326-36.

48. Kawasaki T, Kawai T. Toll-like receptor signaling pathways. Front Immunol. 2014;5:461.

49. Cui YC, et al. The Contribution of Different Components in QiShenYiQi Pills(R) to Its Potential to Modulate Energy Metabolism in Protection of Ischemic Myocardial Injury. Front Physiol. 2018;9:389.

50. Tang DX, et al., QiShenYiQi Pills, a Compound Chinese Medicine, Ameliorates Doxorubicin-Induced Myocardial Structure Damage and Cardiac Dysfunction in Rats. Evid Based Complement Alternat Med, 2013. 2013:

p. 480597.

51. Han JY, et al. Effects and mechanisms of QiShenYiQi pills and major ingredients on myocardial microcirculatory disturbance, cardiac injury and fibrosis induced by ischemia-reperfusion. Pharmacol Res. 2019;147:104386.

52. Despond EA, Dawson JF. Classifying Cardiac Actin Mutations Associated With Hypertrophic Cardiomyopathy. Front Physiol. 2018;9:405.

53. Ye H, et al. Drug Repositioning Through Network Pharmacology. Curr Top Med Chem. 2016;16(30):3646-56.

54. Xue H, et al. Review of Drug Repositioning Approaches and Resources. Int J Biol Sci. 2018;14(10):1232-44.

55. Weng X, et al. An alpha2-adrenoceptor agonist: Dexmedetomidine induces protective cardiomyocyte hypertrophy through mitochondrial-AMPK pathway. Int J Med Sci. 2020;17(16):2454-67.

56. Chen $\mathrm{P}$, et al. Expression and distribution of Src in the nucleus of myocytes in cardiac hypertrophy. Int $\mathrm{J}$ Mol Med. 2013;32(1):165-73.

\section{Figures}




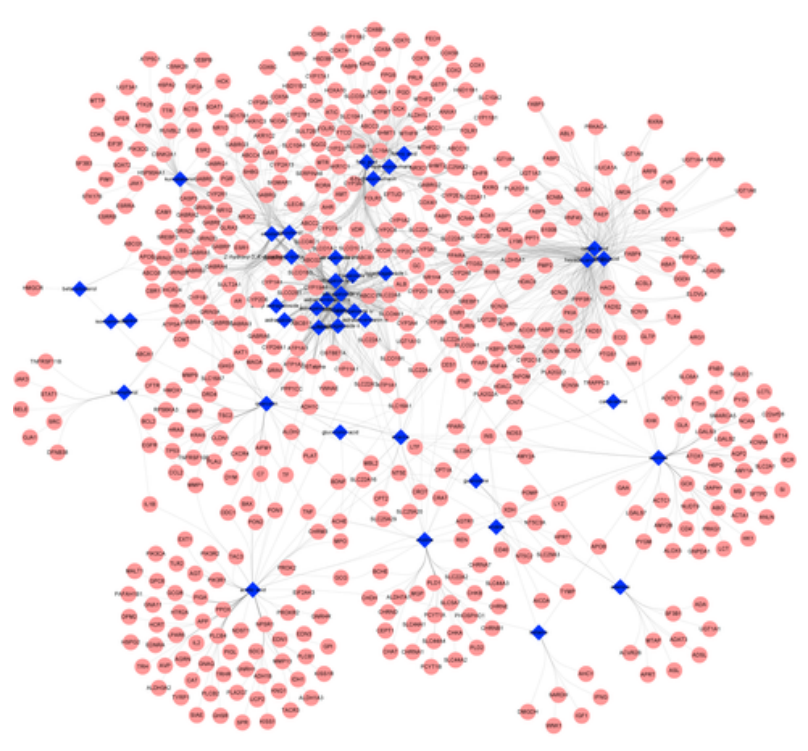

(A) Astragalus membranaceus "Jun" (Emperpor) HQ

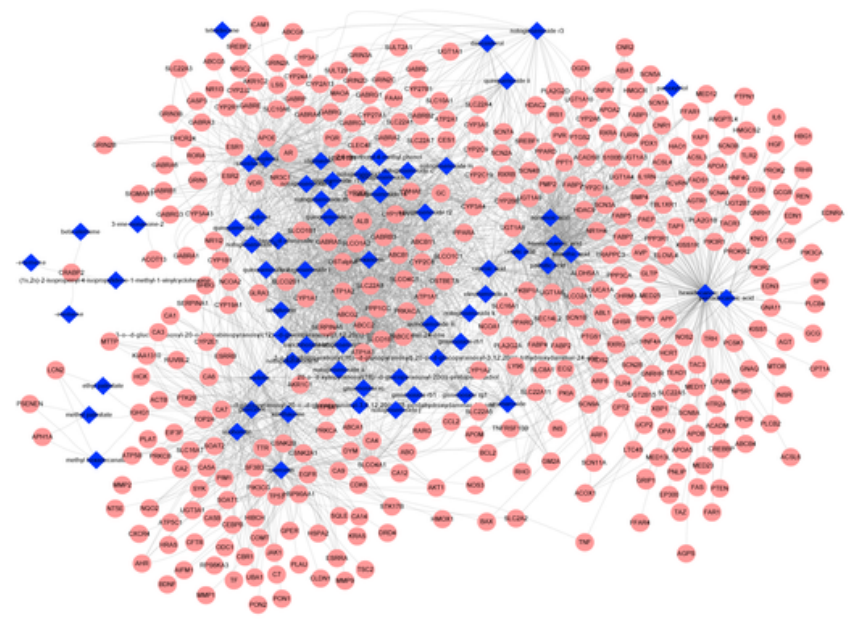

(C) Panax notoginseng "Zuo" (Adjuvant) SQ

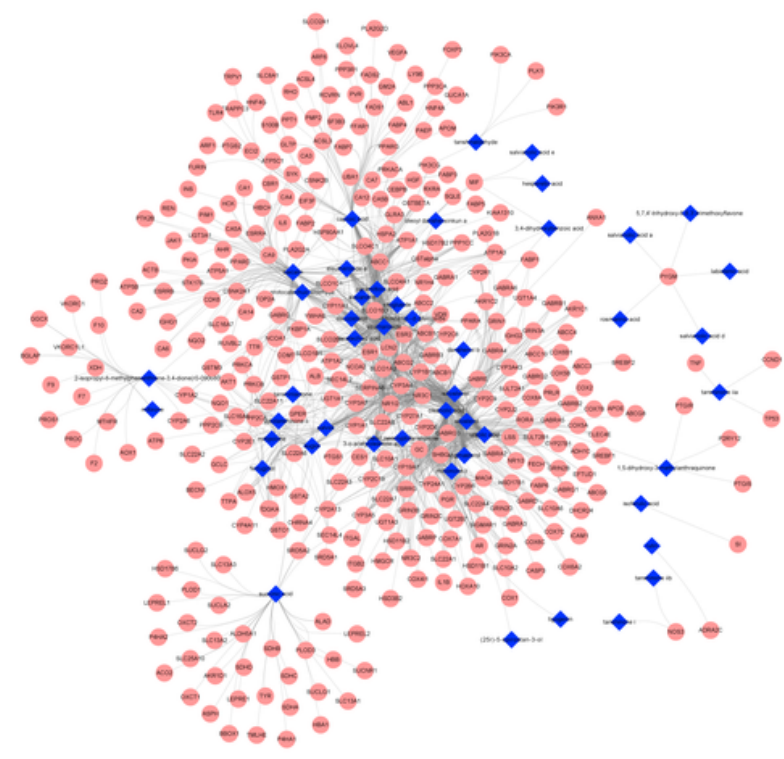

(B) Salvia miltiorrhiza "Chen" (Minister) DS

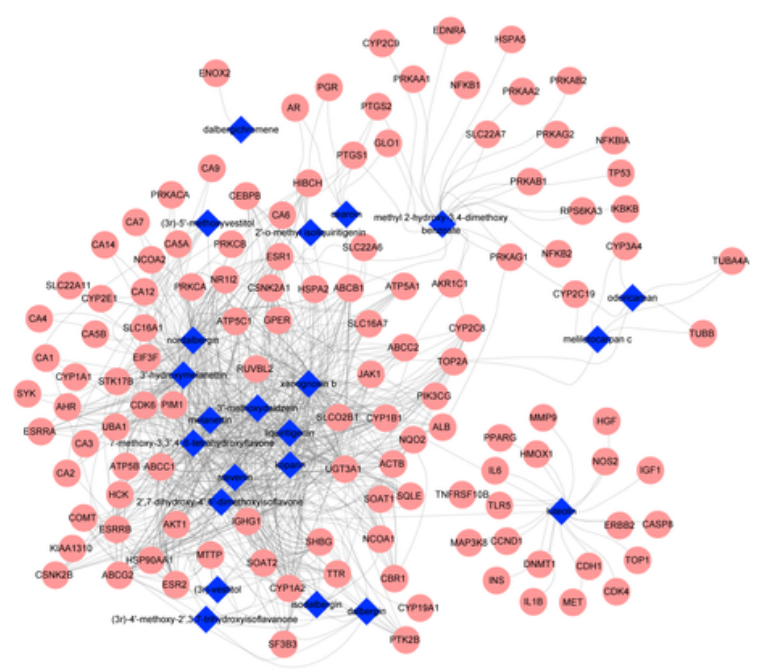

(D) Dalbergia odorifera "Shi" (Courier) JX

Figure 1

The compound-target networks of (A) Astragalus membranaceus, (B) Salvia miltiorrhiza, (C) Panax notoginseng and (D) Dalbergia odorifera in QSYQP. The blue diamond nodes represent compounds, and the pink circle nodes represent targets. 


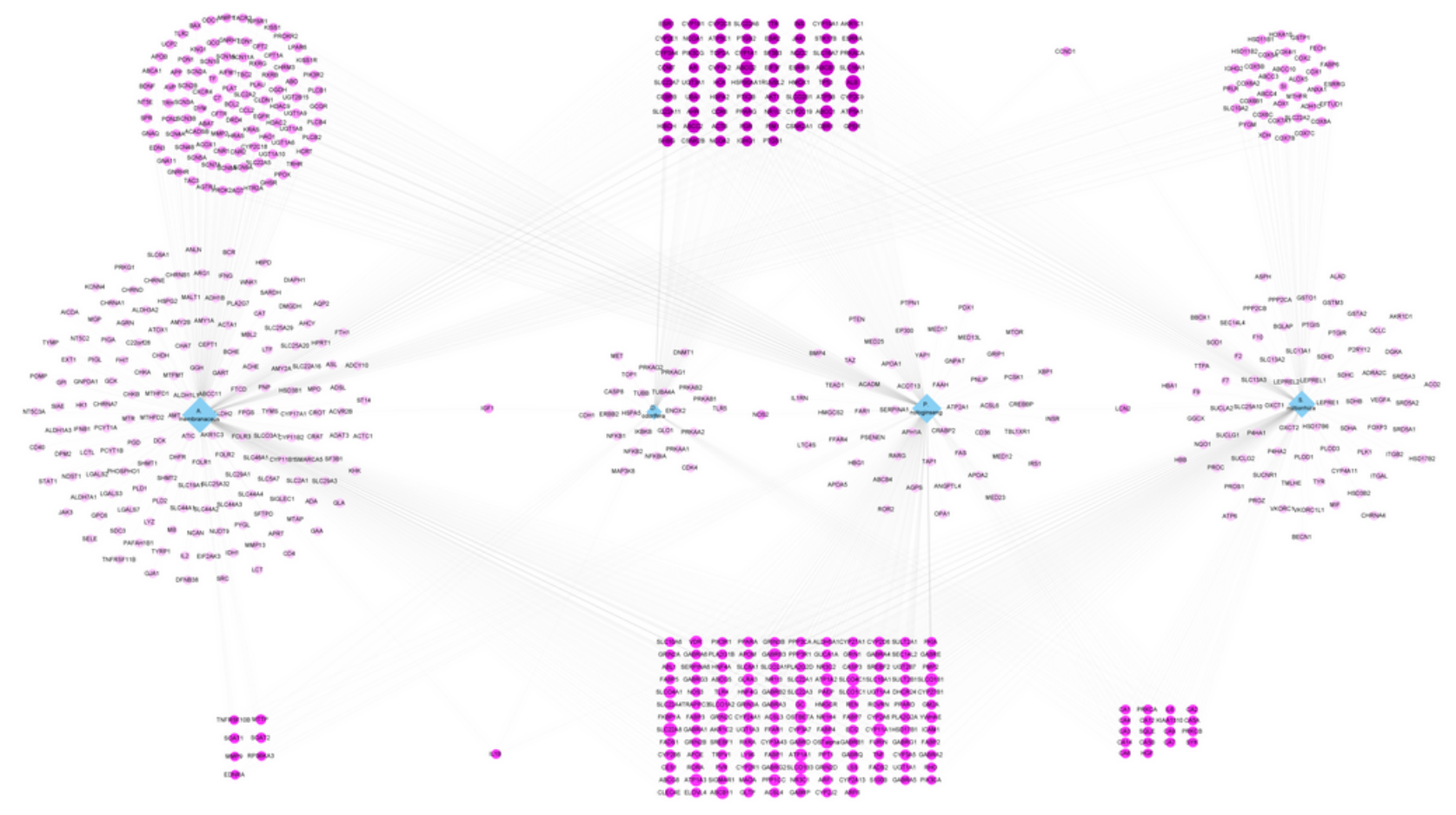

Figure 2

The herb-target network of QSYQP. The diamond nodes represent herbs, and the circle nodes represent targets. The depth of color for a circle node is proportional to the degree of the node, and the size of a circle node is proportional to the number of herb-compound pairs that act on this target.

A. membranaceus

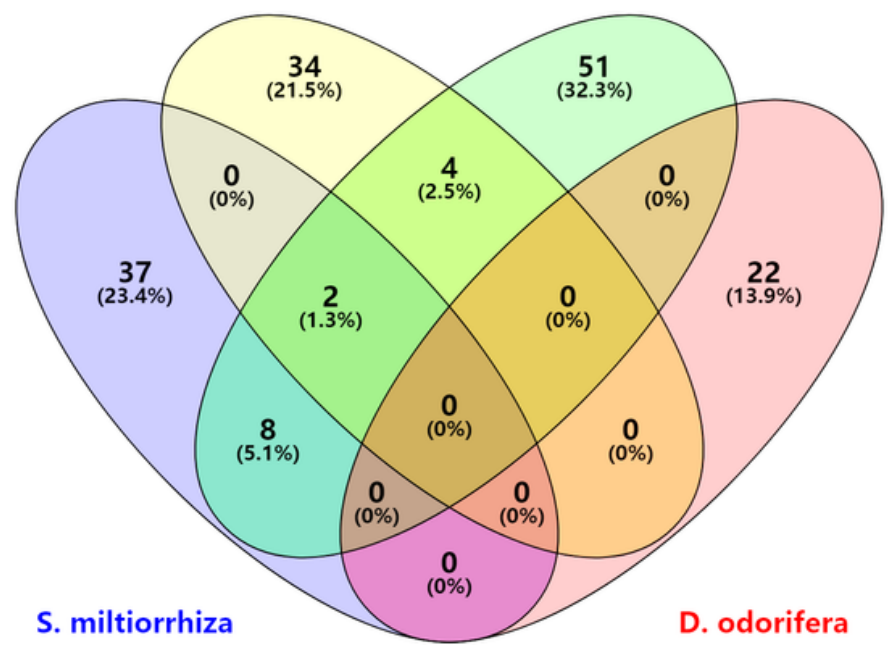

(A)
A. membranaceus

P. notoginseng

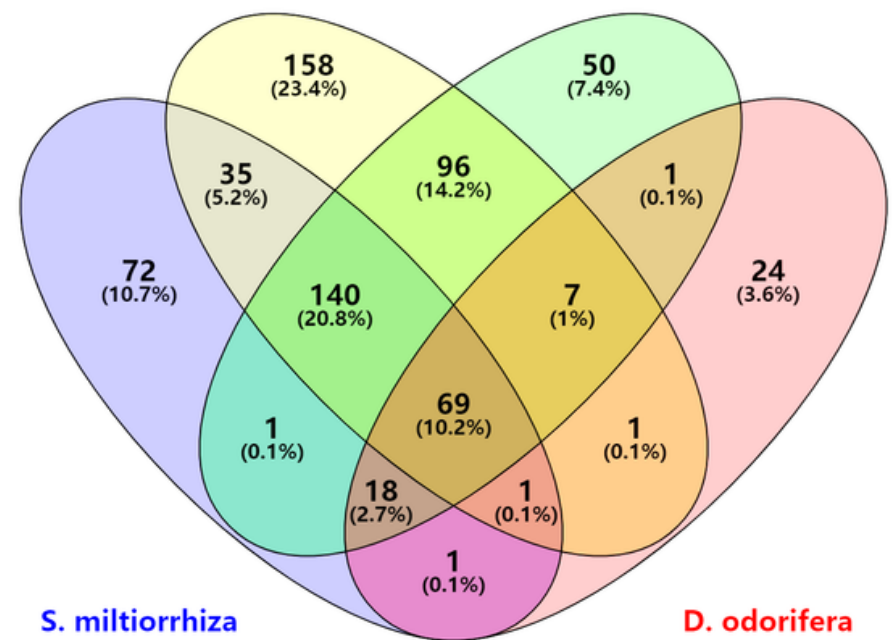

(B)

Figure 3 
Venn diagrams of (A) components and (B) targets of Astragalus membranaceus, Salvia miltiorrhiza, Panax notoginseng and Dalbergiae odorifera in QSYQP. This figure was drawn by free software VENNY 2.120 (https://bioinfogp.cnb.csic.es/tools/venny/index.html).

\section{QSYQP targets \\ CVD genes}

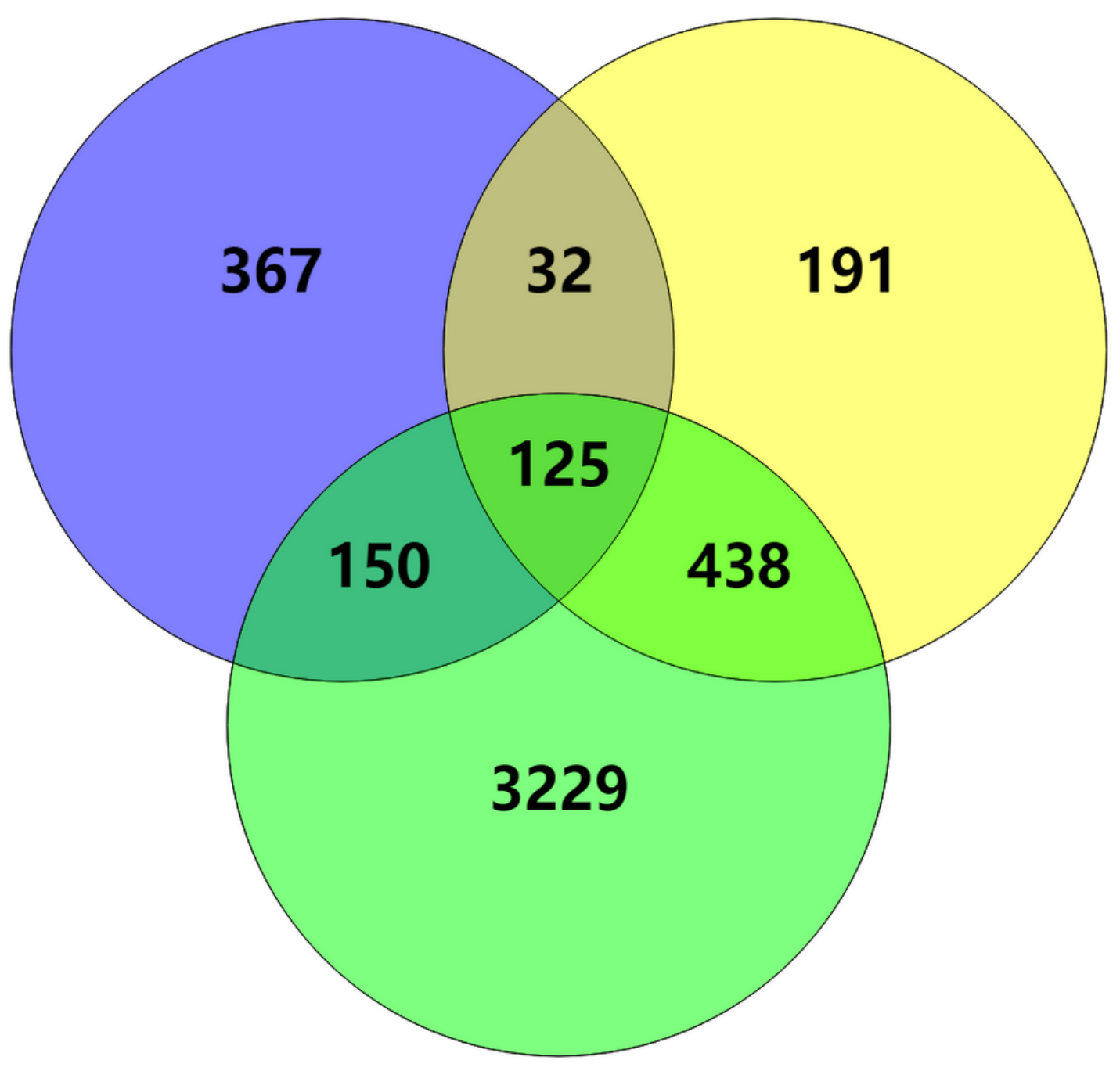

CVD sub-network

Figure 4

Overlap of the targets of QSYQP, and CVD disease genes with nodes in the subnetwork influenced by cardiovascular diseases genes. This figure was drawn by free software VENNY 2.120. 


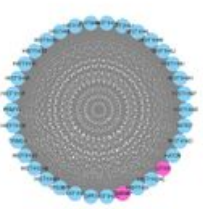

Module 1

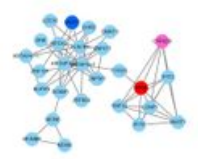

Module 6

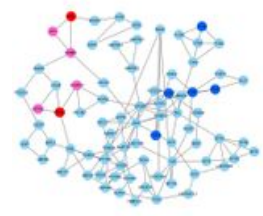

Module 11

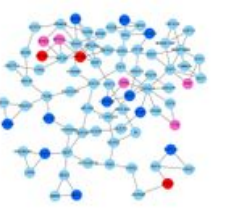

Module 16

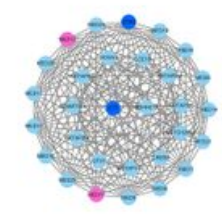

Module 2

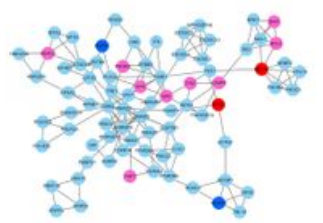

Module 7

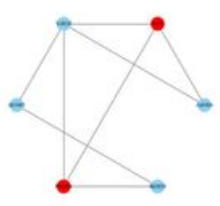

Module 12

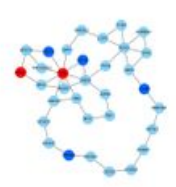

Module 17

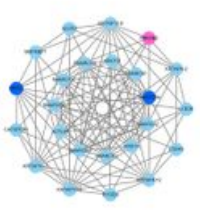

Module 3

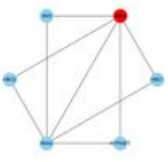

Module 8

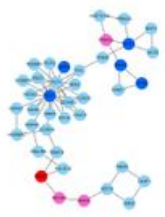

Module 13

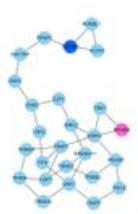

Module 18

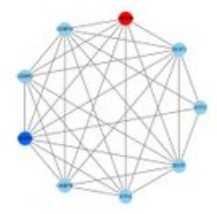

Module 4

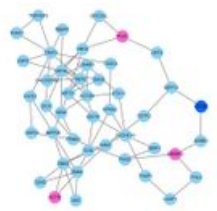

Module 9

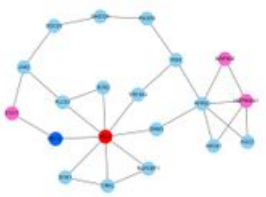

Module 14

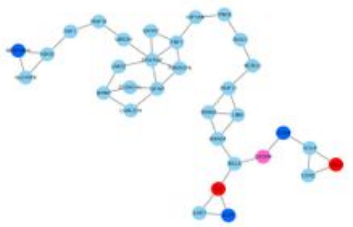

Module 19

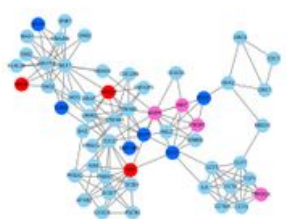

Module 5

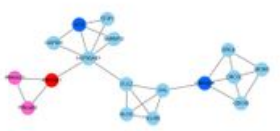

Module 10

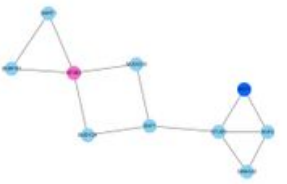

Module 15

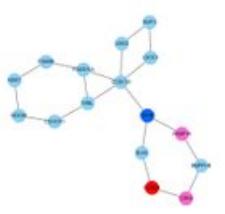

Module 20

\section{Figure 5}

QSYQP acts on 20 modules in the subnetwork influenced by cardiovascular diseases. The dark blue nodes are the disease genes, and the light blue nodes are the genes affected by the diseases. The red and pink nodes are the targets of QSYQP. The red targets are also the genes of cardiovascular diseases. 
A

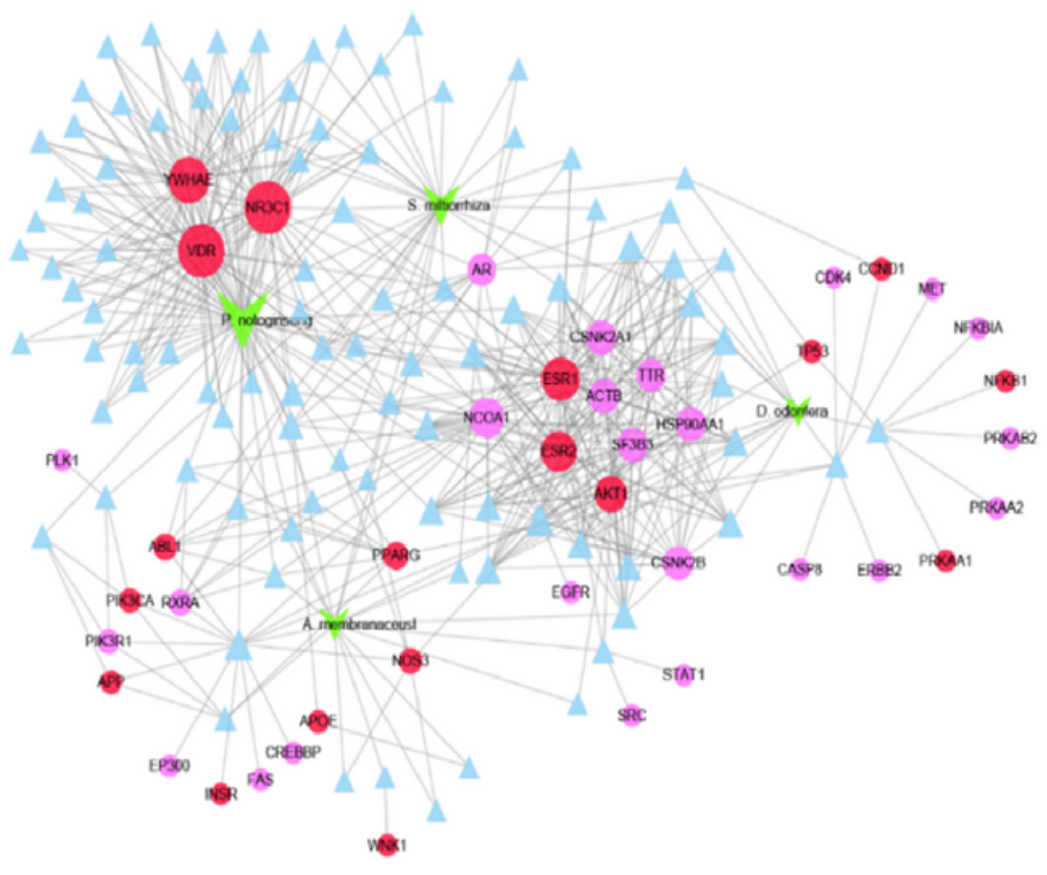

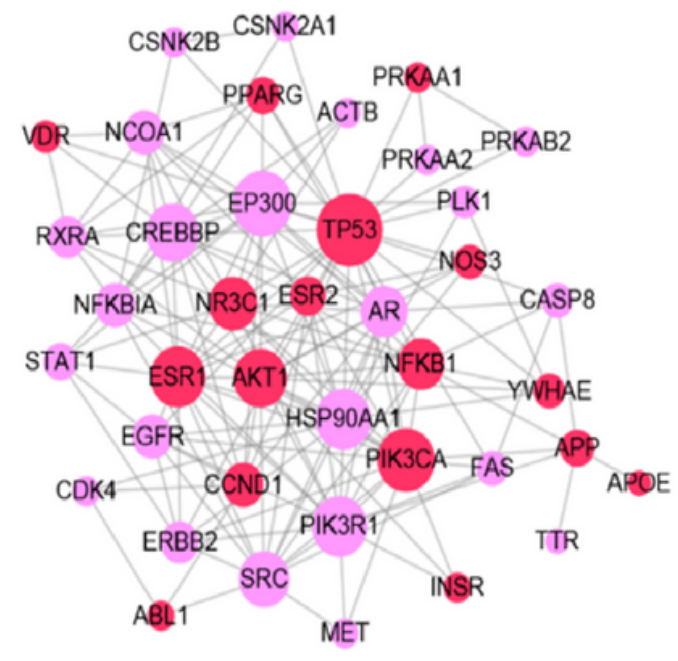

\section{Figure 6}

Network analysis for the important targets of QSYQP. (A) Herb-compound-target network. (B) Protein-protein interaction network. The red circular nodes are the cardiovascular diseases-related genes affected by the formula. The pink circular nodes are the adjacent genes of the cardiovascular diseases-related genes affected by the formula. The green and blue nodes are herbs and compounds of QSYQP, respectively. The size of the node is proportional to its degree in the network. 
A

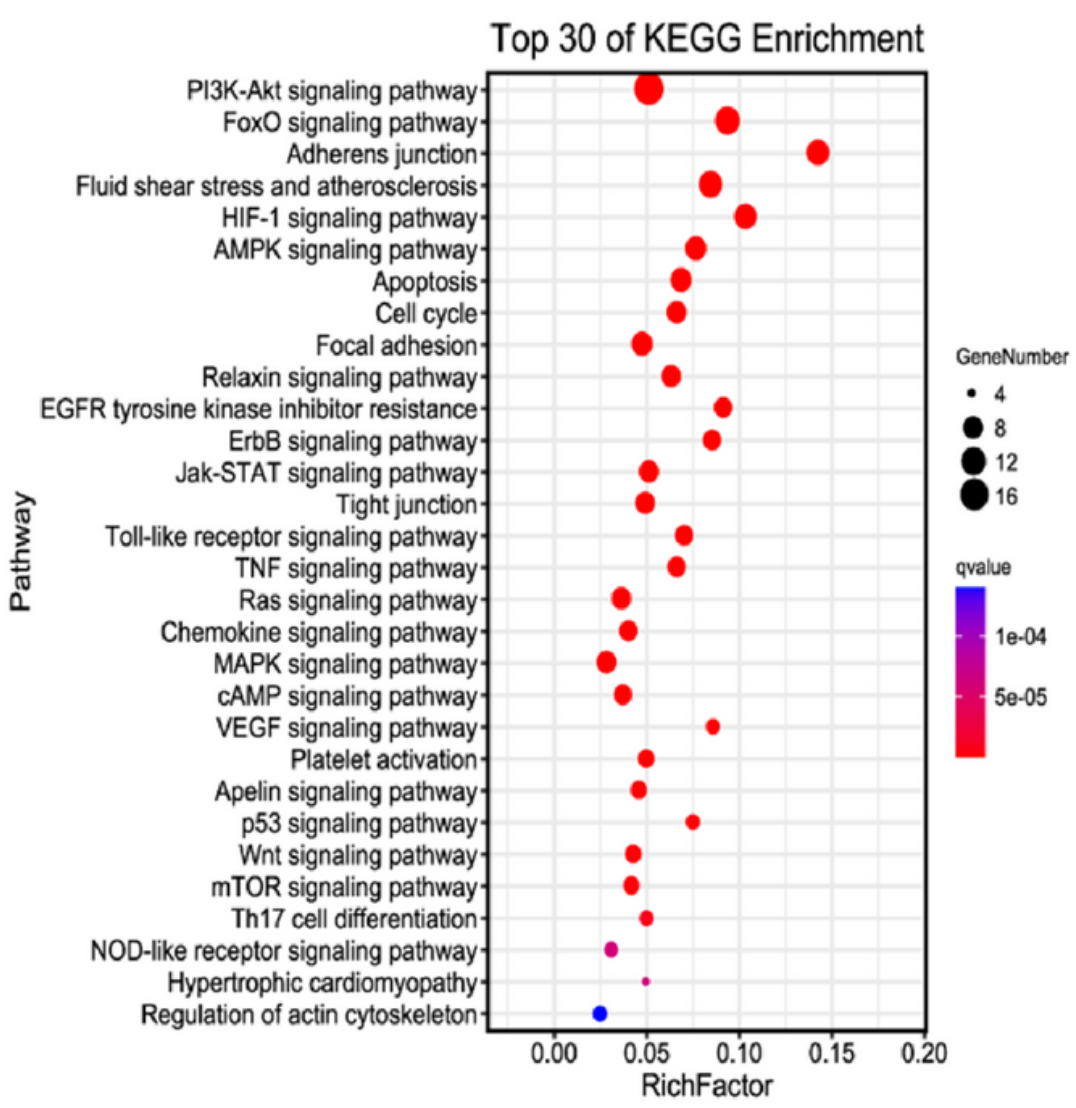

B

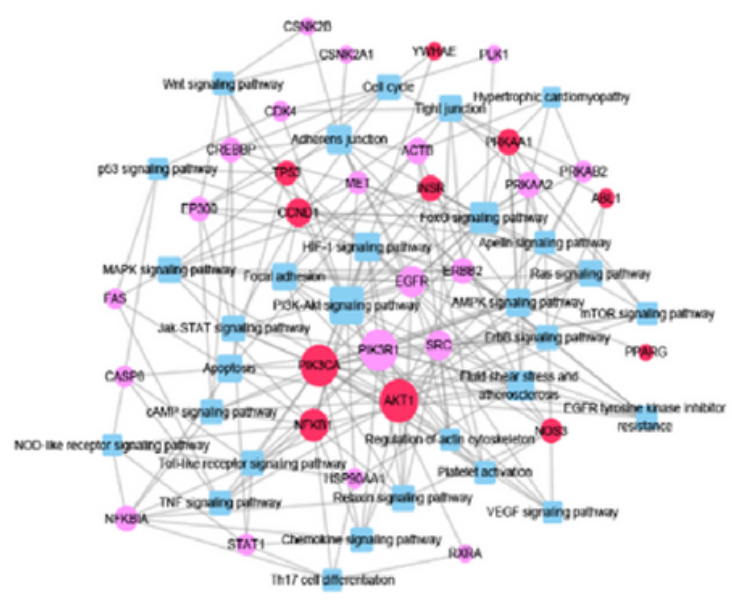

C

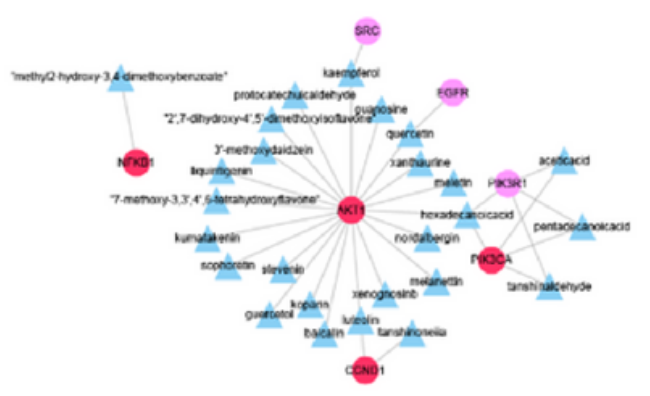

\section{Figure 7}

Pathway analysis for important targets. (A) Bubble chart of the pathways in which the important targets of QSYQP are enriched. (B) Target-pathway network for important targets. (C) compound-target network for key targets of QSYQP identified by network and pathway analysis. The red circular nodes are the cardiovascular diseases-related genes affected by the formula. The pink circular nodes are the adjacent genes of the cardiovascular diseasesrelated genes affected by the formula. 
A

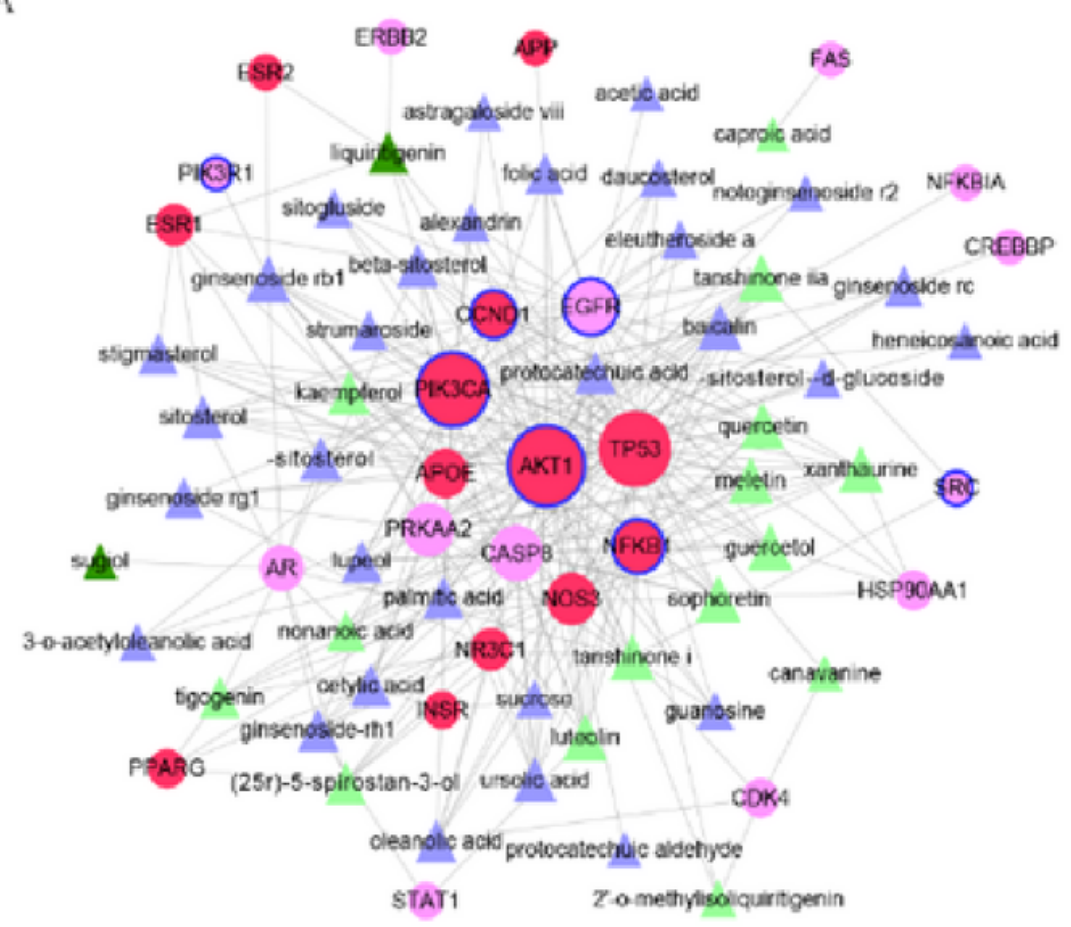

B

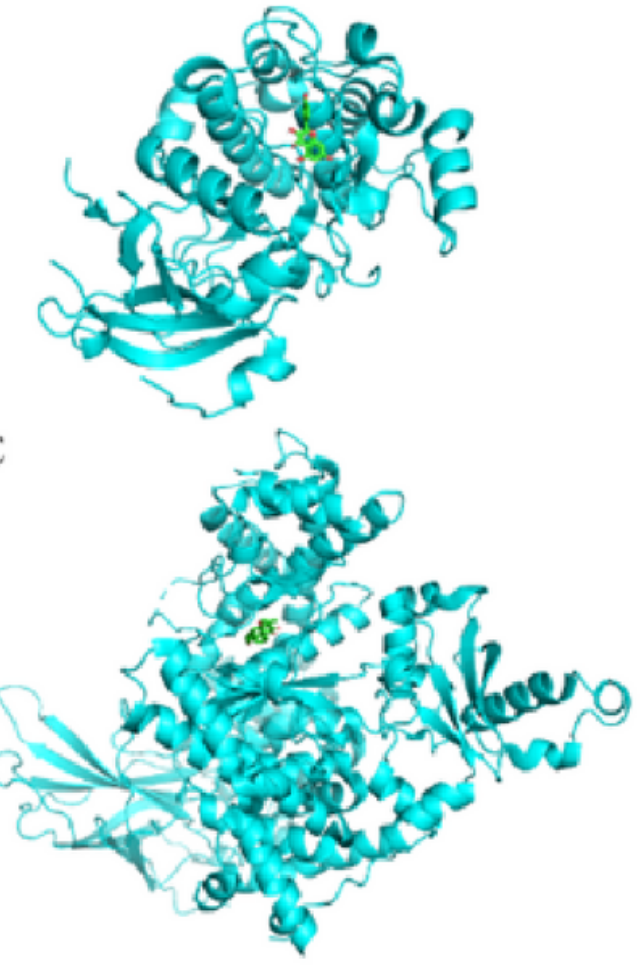

$\mathrm{D}$

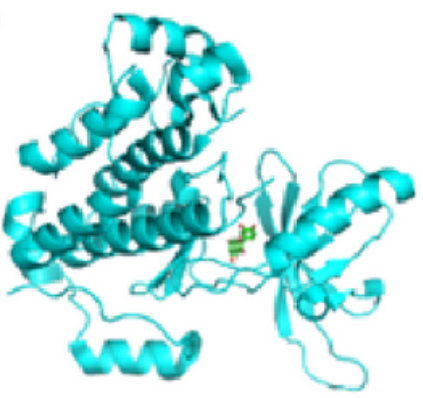

E

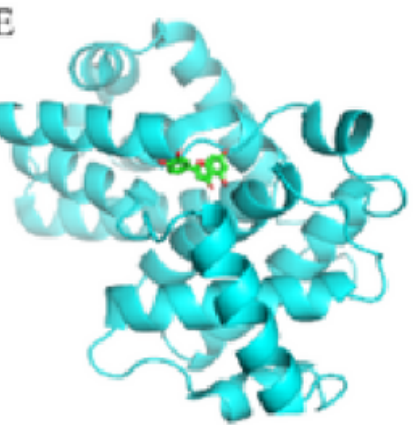

F

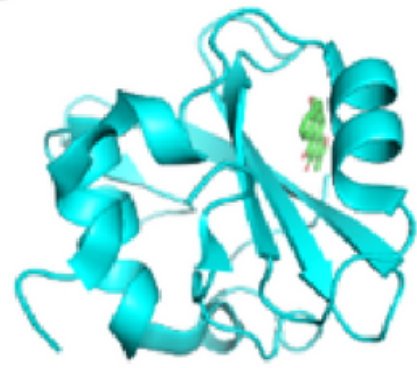

G

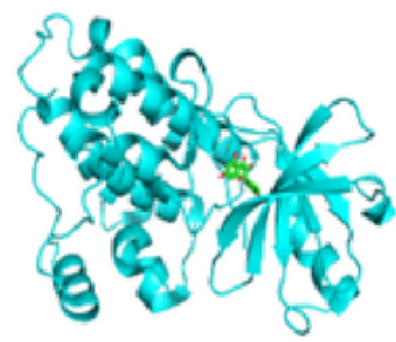

Figure 8

Validation of QSYQP's important targets by literature mining and molecular docking. (A) Compound-target network for the important targets of QSYQP validated by literatures. The red circular nodes are the cardiovascular diseasesrelated genes affected by the formula. The pink circular nodes are the adjacent genes of the cardiovascular diseases-related genes affected by the formula. The dark and shallow green triangles are compounds with good and moderate drug-likeness grading, respectively, and the blue triangles are compounds with weak drug-likeness grading. The size of the circular node is proportional to its degree in the network. (B) (G)Molecular docking of QSYQP's key targets with corresponding compounds. The binding poses of AKT1 complexed with kaempferol (B), PIK3CA complexed with tanshinaldehyde (C), EGFR complexed with quercetin (D), CCND1 complexed with luteolin (E), PIK3R1 complexed with tanshinaldehyde (F), and SRC complexed with kaempferol (G). 
FITC
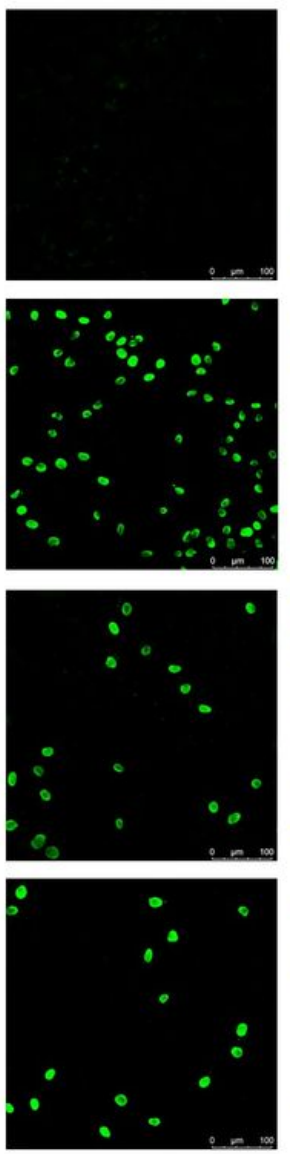

H/R+ASIV

H/R+ASIV+DSS
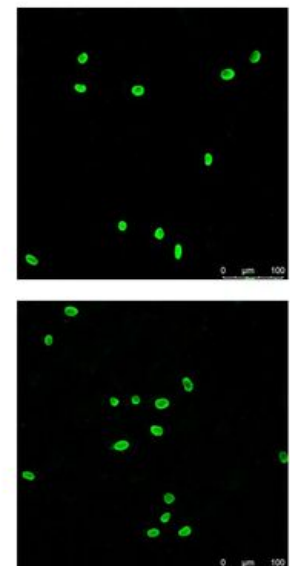

DAPI
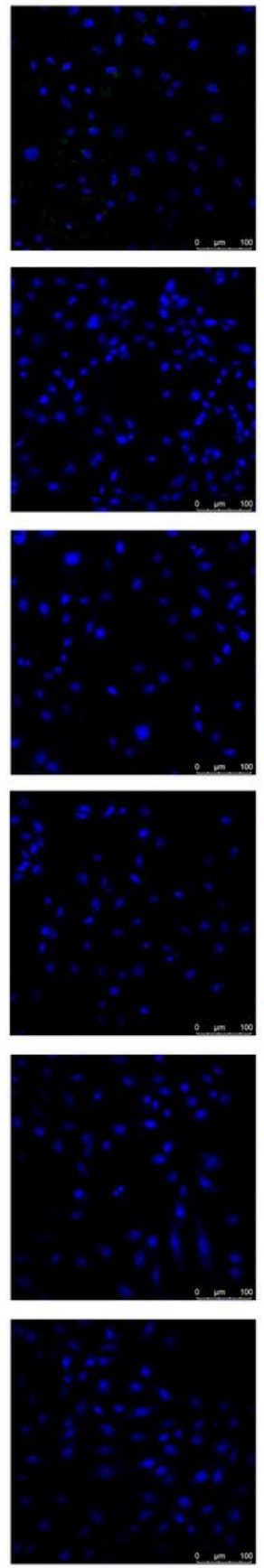

Merge
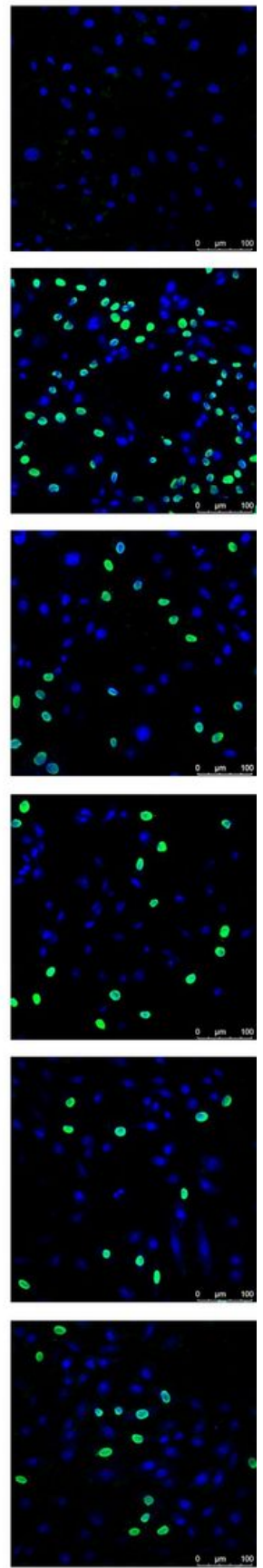

\section{Figure 9}

QSYQP and its main components increased cell survival and rescued H/R injury in $\mathrm{H} 9 \mathrm{c} 2$ cardiomyocytes. The cells were divided into six groups: Control group (Control), hypoxia and reoxygenation group (H/R), H/R+astragaloside IV group (H/R+ASIV), H/R+danshensu group (H/R+DSS), H/R+astragaloside IV and danshensu group (H/R+ASIV+DSS), H/R+Qishen Yiqi Pills group (H/R+QSYQP). Apoptosis cells were identified by FITC staining (green), and total nucleus by DAPI staining (blue). The results showed that QSYQP and its main components significantly prevent apoptosis induced by H/R. Scale bar: $100 \mu \mathrm{m}$. 

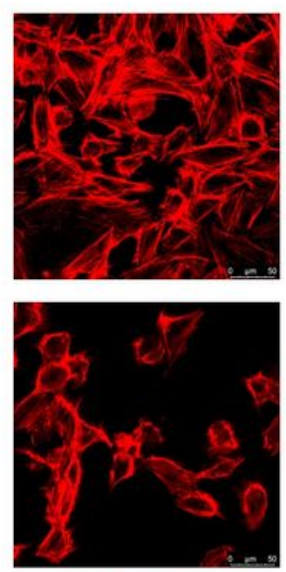

$H / R$

H/R+DSS
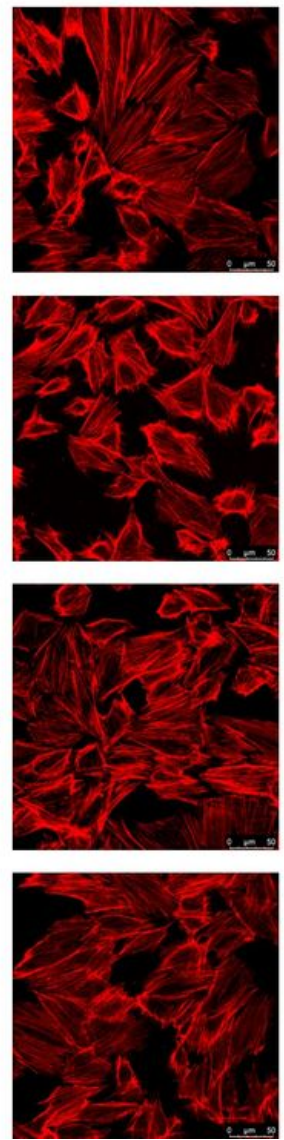
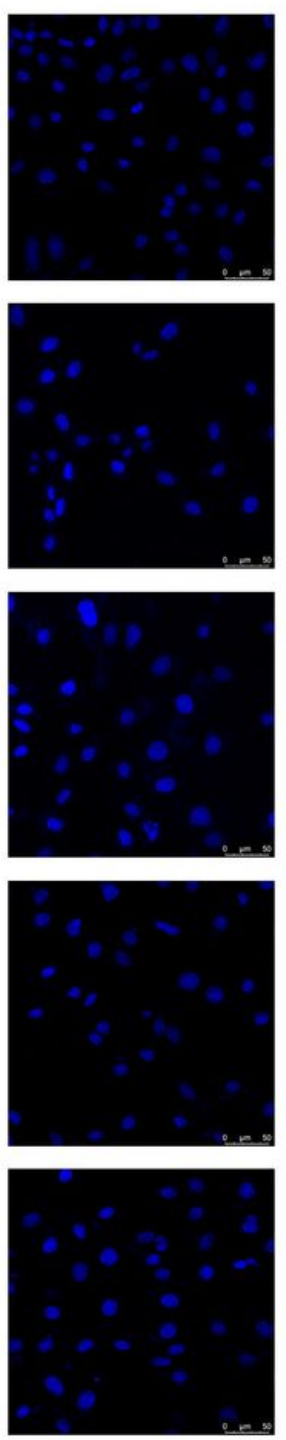

Merge
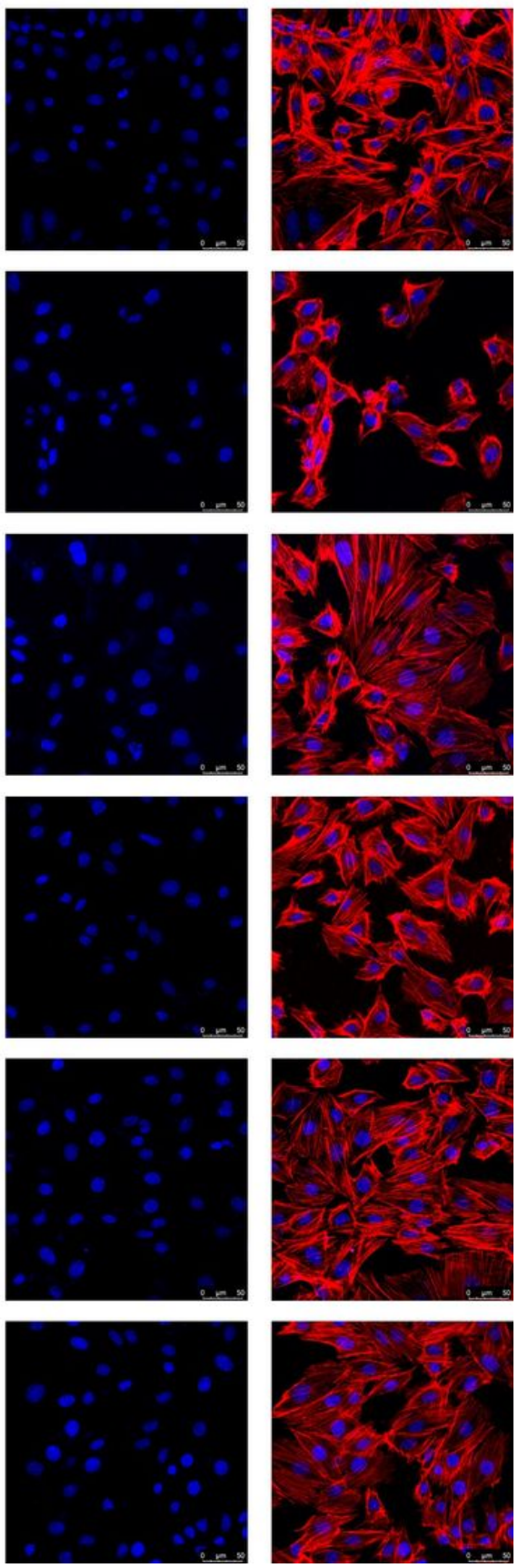

\section{Figure 10}

The effect of QSYQP and its main components on H/R-induced disruption of Cytoskeleton in H9c2 cardiomyocytes. The cells were divided into six groups: Control group (Control), hypoxia and reoxygenation group (H/R),

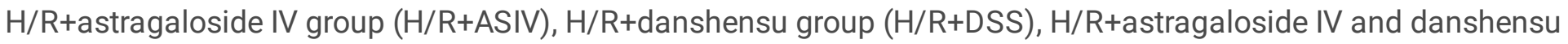
group (H/R+ASIV+DSS), H/R+Qishen Yiqi Pills group (H/R+QSYQP). Compared with the control group, exposure of $\mathrm{H} 9 \mathrm{c} 2$ cardiomyocytes to $\mathrm{H} / \mathrm{R}$ caused a remarkable interrupt and exception aggregation in red fluorescence in the cytoplasm. A massive aggregation and confusion of red fluorescent signals were checked out, nuclei were stained by DAPI (blue). QSYQP significantly reversed the H/R-induced disruption of cytoskeleton. Scale bar: $50 \mu \mathrm{m}$. 
FOXO1

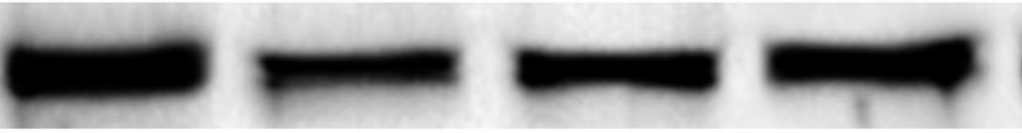

DIAPH1

GAPDH
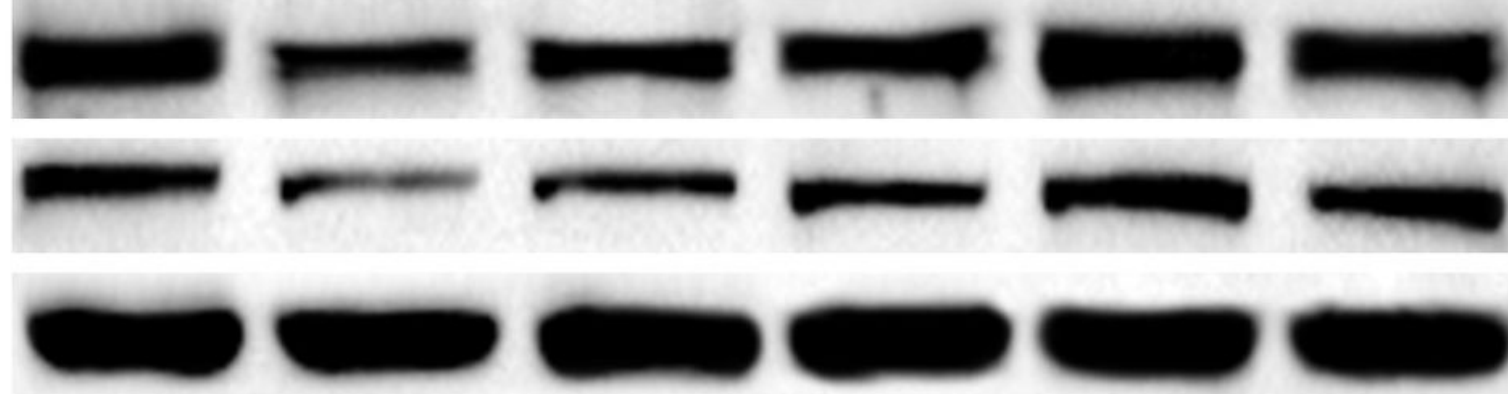

H/R

DSS (400uM/L)

ASIV (400uM/L)

QSYQP $(8 \mathrm{mg} / \mathrm{ml})$

\section{Figure 11}

Expression of ACTC1, FoxO1 and DIAPH1 proteins in $\mathrm{H} 9 \mathrm{c} 2$ cardiomyocytes in each group by Western blotting as described in the Methods. QSYQP treatment induced alterations in key Cytoskeleton-related proteins in $\mathrm{H} 9 \mathrm{c} 2$, including ACTC1, FoxO1 and DIAPH1. Compared with H/R group, the levels of all proteins raised obviously after QSYQP treatment.

\section{Supplementary Files}

This is a list of supplementary files associated with this preprint. Click to download.

- SupplementaryTable1.docx

- SupplementaryTable2.docx

- SupplementaryTable3.docx

- SupplementaryTable4.docx 